\title{
Friedel-Crafts chemistry: Part 41. A new facile synthesis of indeno[1,2-c] pyrazoles, $2 H$-benzo[ $g]$ indazoles and benzo[6,7]cyclohepta[1,2-c]pyrazoles via Friedel-Crafts ring closures
}

\author{
Hassan Abdou Kotb Abd El-Aal *, Ahmed Mahmoud Abdel Mageed El-Khawaga and Ali Ali Khalaf \\ Chemistry Department, Faculty of Science, Assiut University, Assiut, 71516, Egypt \\ *Corresponding author at: Chemistry Department, Faculty of Science, Assiut University, Assiut, 71516, Egypt. \\ Tel.: +2.088.2411485. Fax: +2.088.2342708. E-mail address: hassankotb33@yahoo.com (H.A.K.A. El-Aal).
}

\section{ARTICLE INFORMATION}

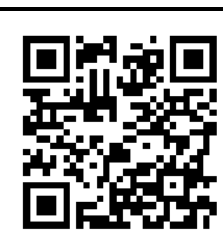

DOI: $10.5155 /$ eurjchem.5.2.277-286.976

Received: 17 November 2013

Received in revised form: 25 December 2013

Accepted: 25 December 2013

Online: 30 June 2014

\section{KEYWORDS}

Benzo[g]indazole

Heteropolycycles

Heteroarylalkanols

Indeno[1,2-c]pyrazole

Friedel-Crafts ring closures

Benzo[6,7]cyclohepta[1,2-c]pyrazole

\section{Introduction}

The synthesis of pyrazoles and their condensed scaffolds have been a subject of consistent interest because of their long history of application in pharmaceutical [1-3] and agrochemical [4-6] industries.

Over the past two decades, pyrazole-containing compounds have received considerable attention owing to their diverse chemotherapeutic potentials. They display a broad spectrum of biological activities such as antimicrobial [7], antifungal [8], anti-inflammatory [9], antitumor [10], antihyperglycemic [11], analgesic [12], antipyretic [13], antibacterial [14], sedativehypnotic [15], antidepressant [16], anti-rheumatoid arthritis [17], anticonvulsant [18], antipsychotic [19], antileukemic [20] and as HIV integrase inhibitor [21] agents. Also pyrazole derivatives were present in numerous herbicides [22], fungicides [23], insecticides [24], pesticides [25] and dyestuffs [26]. In spite of they are rare in nature, the only natural pyrazole (1-pyrazolyl-alanine) was isolated from seeds of watermelons [27].

Although the discovery [28] of pyrazoles as antipyretic agents dated back to 1884, synthesis of pyrazole systems have been a prominent research objective for over a century and a variety of well-established synthetic methodologies are available in the literature. Of the methods developed, 1,3dipolar cycloadditions of diazocompounds [29] or nitrilimines with alkynes [30] or alkenes [31,32], reaction of chalcones and hydrazines [33], reaction of enaminones with different aminoheterocycles [34], condensation of 1,3-diketones and hydrazines in the presence of an acidic catalyst [35] and coupling of terminal alkynes with hydrazine and carbon monoxide [36].

Among different condensed pyrazoles polycycles, indeno[1,2-c]pyrazole (1) [37,38], $2 H$-benzo[g]indazole (2) [39-41] and benzo[6,7]cyclohepta[1,2-c]pyrazole (3) [42] derivatives (Figure 1) are such molecules which have unique nitrogen-containing tricyclic structures are known to exert biological activities in many active pharmaceutical ingredients $[43,44]$.

Literature survey revealed that limited numbers of strategies have been successfully applied in the synthesis of such 1-3 skeletons derivatives. However, all such reported methods were based on the addition of substituted hydrazines to suitable chalcones, $\beta$-diketones and $\alpha$-keto- $\beta$-hydroxyesters.

In an alternative pioneering strategy, Li et al. [45] achieved the synthesis of scaffold $\mathbf{2}$ and variety of aza-polycyclic aromatic compounds via superelectrophilic cyclizations of alkenyl-substituted $\mathrm{N}$-heterocycles with triflic acid $\left(\mathrm{CF}_{3} \mathrm{SO}_{3} \mathrm{H}\right)$. 


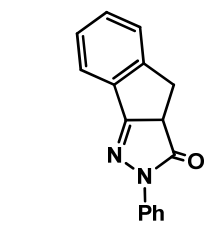

2,4-Dihydro-2-phenylindeno [1,2-c]pyrazole (1)

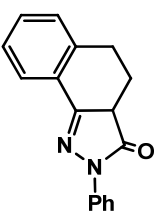

4,5-Dihydro-2-phenyl-2Hbenzo[g]indazole (2)

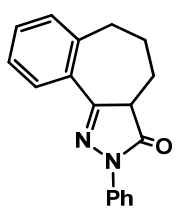

4,5,6-Trihydro-2-phenyl-benzo[6,7] cyclohepta[1,2-c]pyrazole (3)

Figure 1. Pyrazole heteropolycycles containing pharmaceuticals.

The search for new methods for the simple, direct, concise, atom-economical synthetic route and efficient construction of this class of pyrazole compounds still constitutes a major challenge in organic synthesis.

In recent communications [46-50], we have demonstrated simple alternative procedures in the construction of novel and known heterocyclic systems proving the synthetic utility of Friedel-Crafts [51-54] cyclialkylation approach. The results manifested that a number of difficult heterocyclic skeletons can be easily prepared with the advantages of short reaction time and high yield. In view of the above reports and in continuation of our research in Friedel-Crafts ring closures, herein, we wish to report facile and efficient synthesis of substituted indeno[1,2-c]pyrazoles, $2 H$-benzo[g]indazoles and benzo[6,7] cyclohepta[1,2-c]pyrazole via Friedel-Crafts ring closures of heteoraryl acids and alkanols in the presence of both Brönsted and Lewis acid catalysts.

\section{Experimental}

\subsection{Instrumentation}

All reagents were purchased from Merck, Sigma or Aldrich Chemical Co. and were used without further purification. Melting points were measured on a digital Gallenkamp capillary melting point apparatus and are uncorrected. The IR spectra were determined with a Shimadzu 470 infrared spectrophotometer using $\mathrm{KBr}$ wafer and thin film techniques. The ${ }^{1} \mathrm{H}$ and ${ }^{13} \mathrm{C}$ NMR spectra were recorded on Jeol LA $400 \mathrm{MHz}$ FT-NMR (400 MHz for ${ }^{1} \mathrm{H}, 100 \mathrm{MHz}$ for ${ }^{13} \mathrm{C}$ ) and on Varian NMR (90 MHz) spectrometers using $\mathrm{CDCl}_{3}$ or DMSO- $d_{6}$ solvents with TMS as internal standard. Chemical shifts $(\delta)$ and $J$ values are reported in ppm and $\mathrm{Hz}$, respectively. Elemental analyses were performed on a Perkin-Elmer 2400 Series II analyser. The mass spectra were recorded on a JEOL JMS 600 spectrometer at an ionizing potential of $70 \mathrm{eV}$ (EI) electron impact ionization mode using the direct inlet system. Reactions were monitored by thin-layer chromatography (TLC) using pre-coated silica plates and visualized with UV light. Flash column chromatography was performed on silica gel and basic alumina.

\subsection{Synthesis}

\subsubsection{5-Chloro-1,3-diphenyl-1H-pyrazole-4-carbaldehyde (5)}

Pyrazolone 4 ( $4.7 \mathrm{~g}, 0.02 \mathrm{~mol}$ ) was added in small portions over 5 min to an ice-cold Vilsmeier reagent prepared by slowly addition of $\mathrm{POCl}_{3}(9.8 \mathrm{~mL}, 16.12 \mathrm{~g}, 0.1 \mathrm{~mol})$ to DMF (17.8 mL, $16.8 \mathrm{~g}, 0.23 \mathrm{~mol}$ ) at $0{ }^{\circ} \mathrm{C}$ over $30 \mathrm{~min}$. The reaction mixture was heated for $3 \mathrm{~h}$ at $90^{\circ} \mathrm{C}$ then cooled to $0{ }^{\circ} \mathrm{C}$, poured into ice-cold water $(100 \mathrm{~mL})$ and finally treated with potassium carbonate till $\mathrm{pH}=9$. The separated yellow solid was filtered, dried and recrystallized from benzene to afford (4.4 g, $79 \%$ ) of pure aldehyde 5 (Scheme 1). Color: White crystals. M.p.: $107{ }^{\circ} \mathrm{C}$ (Lit. [55], M.p.: 109-111 $\left.{ }^{\circ} \mathrm{C}\right)$. IR ( $\left.\mathrm{KBr}, v, \mathrm{~cm}^{-1}\right): 3110,3065$ (CH) (Aromatic), 2830, $2745 \quad(\mathrm{CH})$ (aldehyde), $1695 \quad(\mathrm{C}=0)$ (aldehyde), 1605, 1590, 1489, 1440 ( $\mathrm{C}=\mathrm{C}$ ) (Aromatic), 1360,
1189, 716, 658. ${ }^{1} \mathrm{H}$ NMR $\left(90 \mathrm{MHz}, \mathrm{CDCl}_{3}, \delta, \mathrm{ppm}\right): 7.20-8.90(\mathrm{~m}$, $10 \mathrm{H}, \mathrm{Ar}-\mathrm{H}), 10.30$ (s, 1H, CHO). MS (EI, $m / z(\%)): 283\left(\mathrm{M}^{+}+1\right.$, 45), $284\left(\mathrm{M}^{+}+2,28\right), 282\left(\mathrm{M}^{+}, 100\right), 254(16), 253$ (25), 248 (15), 220 (18), 77 (86), 51 (92). Anal. calcd. for $\mathrm{C}_{16} \mathrm{H}_{11} \mathrm{ClN}_{2} \mathrm{O}$ : C, 67.96; H, 3.92; N, 9.91. Found: C, 68.25; H, 3.77; N, 9.90\%.

\subsubsection{5-Chloro-1,3-diphenyl-1H-pyrazole-4-carboxylic acid} (6)

To a solution of compound 5 ( $4.2 \mathrm{~g}, 15 \mathrm{mmol})$ in a mixture of $\mathrm{H}_{2} \mathrm{O} / t$-butanol $(1: 1,30 \mathrm{~mL})$ was added a solution of $\mathrm{KMnO}_{4}$ ( $3.3 \mathrm{~g}, 21 \mathrm{mmol}$ ) in $\mathrm{H}_{2} \mathrm{O}(30 \mathrm{~mL})$ at $70-80{ }^{\circ} \mathrm{C}$ over $2 \mathrm{~h}$. Then an aqueous solution of $\mathrm{KOH}(10 \%, 30 \mathrm{~mL})$ was added while stirring until the solution turned alkaline. The mixture was filtered and the clear filtrate was acidified with concentrated hydrochloric acid $(20 \mathrm{~mL}, 30 \%)$ to $\mathrm{pH}=2$. The resulting precipitate was filtered, washed with water, dried and crystallized from ethanol to give ( $3.8 \mathrm{~g}, 85 \%)$ of pure acid 6 (Scheme 1). Color: White crystals. M.p.: $183^{\circ} \mathrm{C}$. IR $\left(\mathrm{KBr}, v, \mathrm{~cm}^{-1}\right)$ : $3440(\mathrm{OH})$ (acid), $3105(\mathrm{CH})$ (Aromatic), 1690(C=0) (acid), 1610, 1585, 1473, 1440 (C=C) (Aromatic), 1360, 1140, 755. ${ }^{1} \mathrm{H}$ NMR (90 MHz, DMSO- $\left.d_{6}, \delta, \mathrm{ppm}\right): 7.60-8.70(\mathrm{~m}, 10 \mathrm{H}, \mathrm{Ar}-\mathrm{H})$, $11.20(\mathrm{~s}, 1 \mathrm{H}, \mathrm{COOH})$. Anal. calcd. for $\mathrm{C}_{16} \mathrm{H}_{11} \mathrm{ClN}_{2} \mathrm{O}_{2}$ : C, 64.33; $\mathrm{H}$, 3.71; N, 9.38. Found; C, 64.52; H, 3.72; N, 9.33\%.

\subsubsection{4-Bromo-1,3-diphenyl-1H-pyrazol-5(4H)-one (9)}

To a an ice-cold solution of pyrazolone 4 (4.7 g, $20 \mathrm{mmol})$ in $\mathrm{AcOH}(30 \mathrm{~mL})$ was added a solution of bromine $(4.3 \mathrm{~g}, 27$ $\mathrm{mmol})$ in $\mathrm{AcOH}(10 \mathrm{~mL})$ over a period of $10 \mathrm{~min}$ at $5-10{ }^{\circ} \mathrm{C}$. The reaction mixture was stirred for $10 \mathrm{~min}$, diluted with water $(100 \mathrm{~mL})$ over 5-10 $\mathrm{min}$ and left to stand in refrigerator for overnight. The solid was filtered, washed with AcOH (20\%) and dried. Crystallization from ethanol gave $(4.6 \mathrm{~g}, 74 \%)$ of pure bromopyrazolone 9 (Scheme 1). Color: Pale yellow needless. M.p.: $117^{\circ} \mathrm{C}$. IR (KBr, v, cm$\left.{ }^{-1}\right): 3090(\mathrm{CH})$ (Aromatic), $2970(\mathrm{CH})$ (pyrazole), 1678 (C=0) (amide), 1593, 1560, 1490, $1455(\mathrm{C}=\mathrm{C})$ (Aromatic), 1340, 1170, 745, 670. ${ }^{1} \mathrm{H}$ NMR $(90 \mathrm{MHz}$, $\left.\mathrm{CDCl}_{3}, \delta, \mathrm{ppm}\right): 4.6(\mathrm{~s}, 1 \mathrm{H}, \mathrm{CH}), 7.45-8.50(\mathrm{~m}, 10 \mathrm{H}, \mathrm{Ar}-\mathrm{H})$. Anal. calcd. for $\mathrm{C}_{15} \mathrm{H}_{11} \mathrm{BrN}_{2} \mathrm{O}$ : C, 57.16; H, 3.52; N, 8.89. Found; C, $57.05 ; \mathrm{H}, 3.55 ; \mathrm{N}, 8.84 \%$.

\subsubsection{General procedure for the synthesis of substituted acids 10a,b}

A solution of 4-bromo-1,3-diphenyl-1H-pyrazol-5(4H)-one (9) (4.7 g, $15 \mathrm{mmol})$ in $30 \mathrm{~mL}$ of dry benzene was added dropwise over 40 minutes to an ice-cold suspension of sodiomalonic ester prepared from sodium $(1.0 \mathrm{~g}, 45 \mathrm{mmol})$ and substituted diethyl malonate $(5.3 \mathrm{~g}, 33 \mathrm{mmol})$ in $30 \mathrm{~mL}$ of absolute ethanol. After complete addition, the reaction mixture was kept in a refrigerator for $24 \mathrm{~h}$ and then refluxed for $4 \mathrm{~h}$. The solvents were evaporated and the resulting ester was refluxed for $2 \mathrm{~h}$ with $\mathrm{KOH}$ solution $(25 \mathrm{~mL}, 30 \%)$. The mixture was diluted with water $(30 \mathrm{~mL})$, refluxed for an additional $1 \mathrm{~h}$, cooled and acidified with $\mathrm{HCl}$ solution (40 mL, $30 \%)$. The crude substituted malonic acid was filtered, washed with water and dried. 


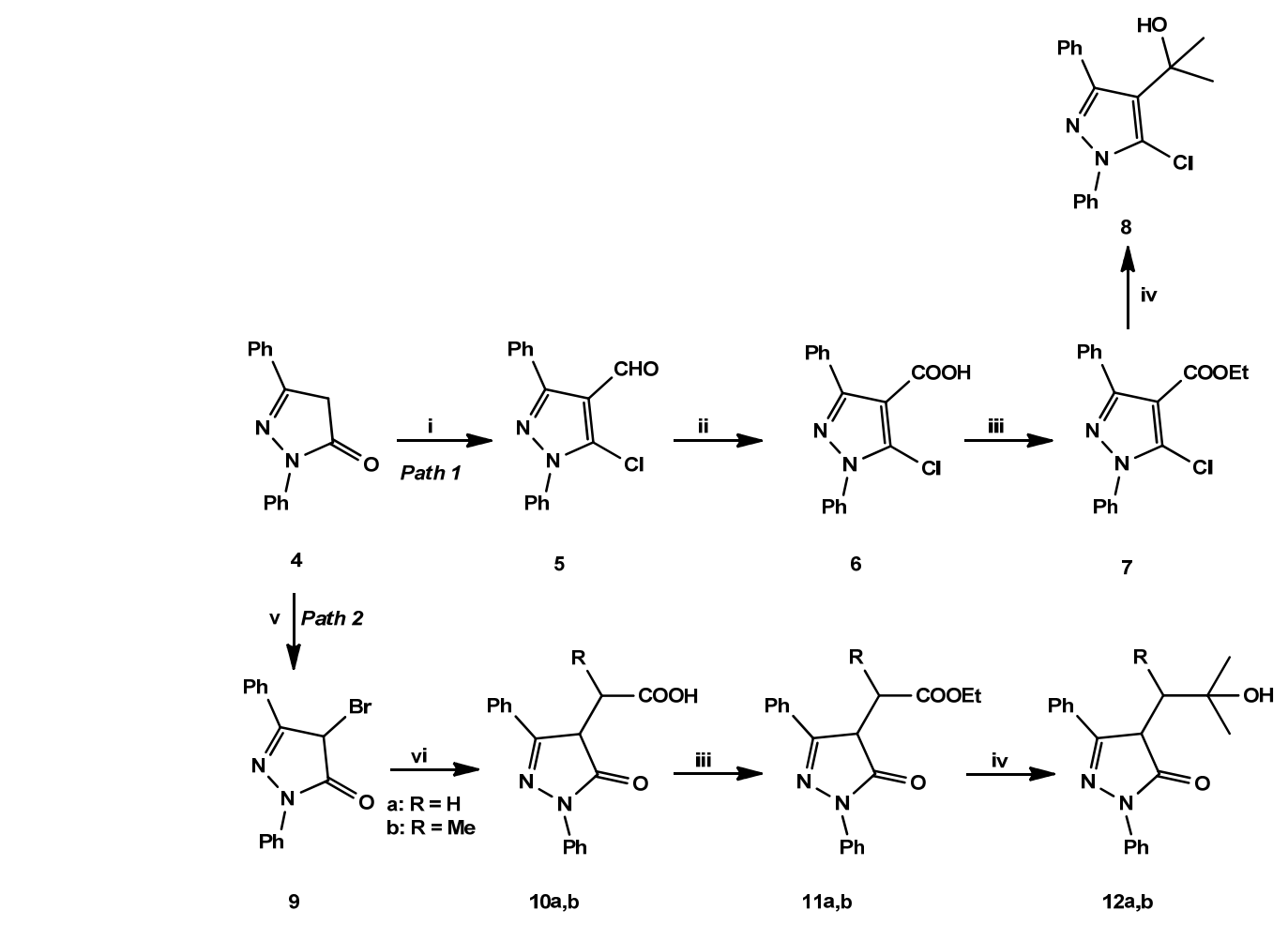

Reagents and conditions: (i) $\mathrm{POCl}_{3} / \mathrm{DMF}, 90^{\circ} \mathrm{C}, 3 \mathrm{~h}, \mathrm{~K}_{2} \mathrm{CO}_{3}$, (ii) $\mathrm{KMnO}_{4}, \mathrm{H}_{2} \mathrm{O} / \mathrm{t}$-butanol, $70-80{ }^{\circ} \mathrm{C}, 2 \mathrm{~h}$, (iii) $\mathrm{EtOH} \mathrm{H}_{2} \mathrm{SO}_{4}, 8 \mathrm{~h}, \mathrm{reflux}, \mathrm{Na}_{2} \mathrm{CO}_{3}$, (iv) $\mathrm{MeMgI}$, THF/Et $2 \mathrm{O}, \mathrm{rt}$, $\mathrm{NH}_{4} \mathrm{Cl}$ soln (Table 1), (v) $\mathrm{Br}_{2} / \mathrm{AcOH}, 5-10{ }^{\circ} \mathrm{C}, 20 \mathrm{~min}$, (vi) (a) $\mathrm{NaCH}(\mathrm{COOEt})_{2}, \mathrm{KOH}, 24 \mathrm{~h}$, (b) heated at 190-200 ${ }^{\circ} \mathrm{C}$.

Scheme 1

The acid was then heated at $190-200^{\circ} \mathrm{C}$ in an oil bath with stirring for 20 minutes and the melt was poured into $20 \mathrm{ml}$ of acetone. The acetone solution was treated with charcoal, warmed, filtered and the solvent was evaporated to afford crude acids. Crystallization from suitable solvents gave pure acids 10a,b (Scheme 1). Analytical, physical and spectroscopic data are given in the following:

2-(4,5-Dihydro-5-oxo-1,3-diphenyl-1H-pyrazol-4-yl)acetic acid (10a): Color: Pale yellow crystals. Yield: $82 \%$. M.p.: $124{ }^{\circ} \mathrm{C}$ (benzene). IR (KBr, v, cm-1): $3370(\mathrm{OH})$ (acid), $3085(\mathrm{CH})$ (Aromatic), $2950(\mathrm{CH})$ (alkyl), $1720(\mathrm{C}=0)$ (acid), $1674(\mathrm{C}=0)$ (amide), 1610, 1590, 1460, 1445 (C=C) (Aromatic), 1335, 1180, 745. ${ }^{1} \mathrm{H} \mathrm{NMR}\left(90 \mathrm{MHz}^{-\mathrm{CDCl}_{3}, \delta}, \mathrm{ppm}\right): 2.61(\mathrm{t}, 1 \mathrm{Ha}, J=14.2 \mathrm{~Hz}$, $\mathrm{CH}_{2}$ ), 2.75 (t, 1Hb, $J=14.2 \mathrm{~Hz}, \mathrm{CH}_{2}$ ), 2.82 (app. t, $1 \mathrm{H}, J=10.4 \mathrm{~Hz}$, $\mathrm{CH})$, 7.30-8.55 (m, 10H, Ar-H), 10.9 (s, 1H, COOH). Anal. calcd. for $\mathrm{C}_{17} \mathrm{H}_{14} \mathrm{~N}_{2} \mathrm{O}_{3}$ : C, 69.38; $\mathrm{H}, 4.79 ; \mathrm{N}$, 9.52. Found; C, 69.25; H, $4.56 ; \mathrm{N}, 9.50 \%$.

2-(4,5-Dihydro-5-oxo-1,3-diphenyl-1H-pyrazol-4-yl) propanoic acid (10b): Color: White crystals. Yield: 75\%. M.p.: $95{ }^{\circ} \mathrm{C}$ (ethanol). IR (KBr, v, cm-1): 3355 (OH) (acid), $3075(\mathrm{CH})$ (Aromatic), 2977(CH) (alkyl), 1715 (C=0) (acid), $1664(\mathrm{C}=0)$ (amide), 1600, 1585, 1450, 1440, 1380, 1130, 749. ${ }^{1} \mathrm{H}$ NMR (90 $\left.\mathrm{MHz}_{\mathrm{CDCl}}, \delta, \mathrm{ppm}\right): 1.24\left(\mathrm{~d}, 3 \mathrm{H}, J=9.4 \mathrm{~Hz}, \mathrm{CH}_{3}\right), 2.92(\mathrm{p}, 1 \mathrm{H}, J=$ $12.4 \mathrm{~Hz}, \mathrm{CH}$ ), 3.04 (app. q, $1 \mathrm{H}, J=12.4 \mathrm{~Hz}, \mathrm{CH}), 7.35-8.23$ (m, 10 $\mathrm{H}, \mathrm{Ar}-\mathrm{H}), 11.6(\mathrm{~s}, 1 \mathrm{H}, \mathrm{COOH})$. Anal. calcd. for $\mathrm{C}_{18} \mathrm{H}_{16} \mathrm{~N}_{2} \mathrm{O}_{3}: \mathrm{C}$, 70.12; H, 5.23; N, 9.09. Found; C, 70.05; H, 5.22; N, 9.26\%.

\subsubsection{General procedure for the synthesis of acids $13 a, b$}

A mixture of aldehyde 5 (4.2 g, $15 \mathrm{mmol})$, acid anhydride (18 mmol) and sodium salt of the corresponding acid (18 mmol) was heated in an oil bath at $140-150{ }^{\circ} \mathrm{C}$ with occasionally stirring for $10-12 \mathrm{~h}$. The warm mixture was poured with stirring into water $(50 \mathrm{~mL})$, basified with $\mathrm{Na}_{2} \mathrm{CO}_{3}$ solution $(20 \mathrm{~mL}, 30 \%)$ and extracted with ether $(2 \times 20 \mathrm{~mL})$ The ether extracts was discarded and the resulting solution was heated with decolorizing carbon $(1 \mathrm{~g})$, filtered while hot then it was poured with stirring into ice-cold concentrated hydrochloric acid chopped with ice $(50 \mathrm{~mL})$. After standing for overnight, the precipitated acids 13a or $\mathbf{b}$ was filtered, washed successively with water and left to dry (Scheme 2). Purifications, yields and spectral data are given in the following:

(E)-3-(5-Chloro-1,3-diphenyl-1H-pyrazol-4-yl)acrylic acid (13a): Color: White crystals. Yield: $74 \%$. M.p.: $156^{\circ} \mathrm{C}$ (acetone). IR (KBr, v, cm-1): $3410(\mathrm{OH})$ (br, acid), $3105(=\mathrm{CH})$ (Alkene), $3060(\mathrm{CH})$ (Aromatic), $2995(\mathrm{CH})$ (alkyl), 1697 (C=0) (acid), $1600(\mathrm{C}=\mathrm{C})$ (Alkene), 1580, 1463, $1440(\mathrm{C}=\mathrm{C})$ (Aromatic), 1360, $1140,745 .{ }^{1} \mathrm{H}$ NMR $\left(90 \mathrm{MHz}, \mathrm{DMSO}-d_{6}, \delta, \mathrm{ppm}\right): 6.35(\mathrm{~d}, 1 \mathrm{H}, J=$ $16.4 \mathrm{~Hz},=\mathrm{CH}), 7.20-8.10(\mathrm{~m}, 10 \mathrm{H}, \mathrm{Ar}-\mathrm{H}), 8.31(\mathrm{~d}, 1 \mathrm{H}, J=16.4$ $\mathrm{Hz},=\mathrm{CH}), 11.2(\mathrm{~s}, 1 \mathrm{H}, \mathrm{COOH})$. Anal. calcd. for $\mathrm{C}_{18} \mathrm{H}_{13} \mathrm{ClN}_{2} \mathrm{O}_{2}: \mathrm{C}$, 66.57; H, 4.03; N, 8.62. Found; C, 66.49; H, 4.22; N, 8.64\%.

(E)-3-(5-Chloro-1,3-diphenyl-1H-pyrazol-4-yl)-2-methyl acrylic acid (13b): Color: Pale yellow solid. Yield: 82\%. M.p.: $172{ }^{\circ} \mathrm{C}$ (ethanol). IR (KBr, v, cm-1): $3410(\mathrm{OH})$ (br, acid), 3094 $(=\mathrm{CH})$ (Alkene), $3024(\mathrm{CH})$ (Aromatic), $2975(\mathrm{CH})$ (alkyl), 1700 $(\mathrm{C}=0)$ (acid), 1622 (C=C) (Alkene), 1588, 1450, 1440 (C=C) (Aromatic), 1385, 1145, 749. ${ }^{1} \mathrm{H}$ NMR $\left(90 \mathrm{MHz}, \mathrm{DMSO}-d_{6}, \delta\right.$, ppm): $1.83\left(\mathrm{~s}, 3 \mathrm{H}, \mathrm{CH}_{3}\right), 7.20-8.36(11 \mathrm{H}, \mathrm{m}, \mathrm{Ar}-\mathrm{H},=\mathrm{CH}), 10.9$ $(1 \mathrm{H}, \mathrm{s}, \mathrm{COOH})$. Anal. calcd. for $\mathrm{C}_{19} \mathrm{H}_{15} \mathrm{ClN}_{2} \mathrm{O}_{2}$ : C, 67.36; $\mathrm{H}, 4.46 ; \mathrm{N}$, 8.27. Found; C, $67.30 ; \mathrm{H}, 4.55 ; \mathrm{N}, 8.40 \%$.

\subsubsection{General procedure for reduction of acids $14 a, b$}

To a solution of acid 13a or $\mathbf{1 3 b}(10 \mathrm{mmol})$ in sodium hydroxide solution $(15 \mathrm{~mL}, 1 \mathrm{~N})$ was treated with sodium amalgam $(25 \mathrm{~g}, 2.5 \%)$ in small portions over a period of $20 \mathrm{~min}$ with vigorous stirring. 


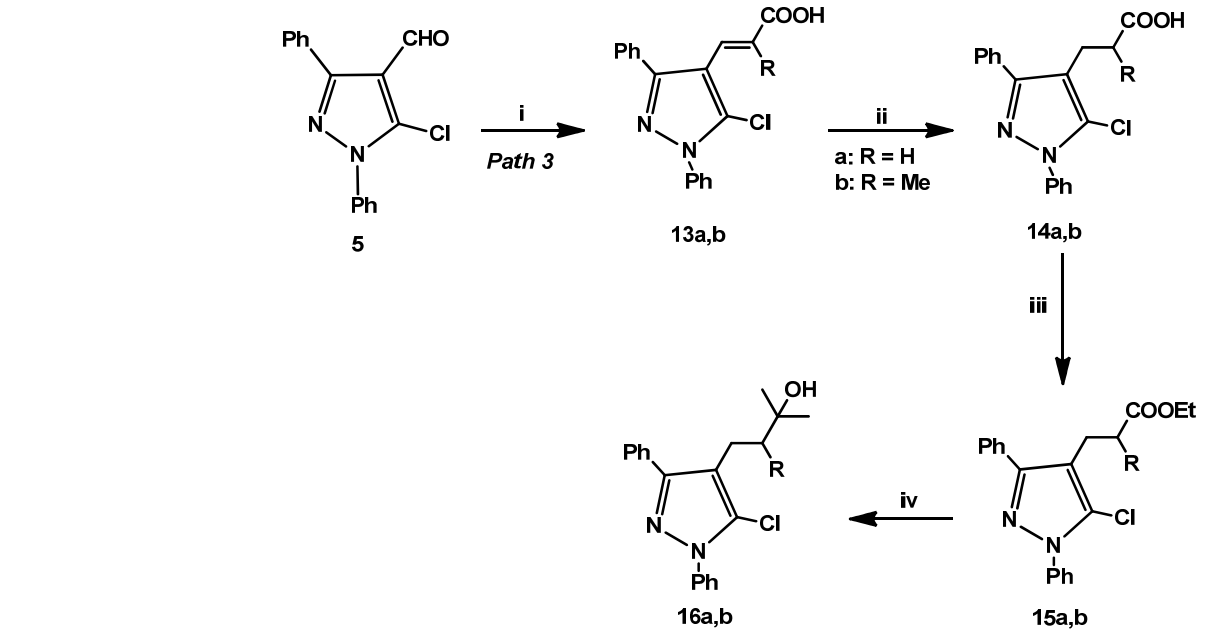

Reagents and conditions: (i) ( $\left.\mathrm{Ac}_{2} \mathrm{O} / \mathrm{AcONa}\right)$ or $\left(\mathrm{Pr}_{2} \mathrm{O} / \mathrm{PrONa}\right) / 140-150{ }^{\circ} \mathrm{C}, \mathrm{Na}_{2} \mathrm{CO}_{3}, 10-12 \mathrm{~h}$, (ii) $\mathrm{Na} / \mathrm{Hg}\left(2.5 \%\right.$ ), $\mathrm{NaOH}, 5 \mathrm{~h},(\mathrm{iii}) \mathrm{EtOH} / \mathrm{H}_{2} \mathrm{SO} \mathrm{H}_{4}, 8 \mathrm{~h}, \mathrm{reflux}$, $\mathrm{Na}_{2} \mathrm{CO}_{3}$, (iv) MeMgI, THF/Et $2 \mathrm{O}, \mathrm{rt}, \mathrm{NH}_{4} \mathrm{Cl}$ solution (Table 1).

Scheme 2

The reaction mixture was stirred for additional $5 \mathrm{~h}$ at room temperature and then mercury was separated, washed with water and the washings were added to the main solution. The whole solution was acidified with concentrated $\mathrm{HCl}(10 \mathrm{~mL})$ and the crude acid 14a or b was filtered, washed with water and dried (Scheme 2). Yields and spectral data are given in the following:

3-(5-Chloro-1,3-diphenyl-1H-pyrazol-4-yl)propanoic acid (14a): Color: White needles. Yield: $92 \%$. M.p.: $135{ }^{\circ} \mathrm{C}$ (ethanol). IR (KBr, v, cm-1): $3345(\mathrm{OH})$ (br, acid), 3090(CH) (Aromatic), 2988(CH) (alkyl), 2490, 1720 (C=0) (acid), 1605, 1590, 1465, $1450(\mathrm{C}=\mathrm{C})$ (Aromatic), 1350, 1165, 745. ${ }^{1} \mathrm{H}$ NMR $(90 \mathrm{MHz}$, $\left.\mathrm{CDCl}_{3}, \delta, \mathrm{ppm}\right): 2.65\left(\mathrm{t}, 2 \mathrm{H}, J=6.2 \mathrm{~Hz}, \mathrm{CH}_{2}\right), 2.90(\mathrm{t}, 2 \mathrm{H}, J=6.2$ $\left.\mathrm{Hz}, \mathrm{CH}_{2}\right), 6.83-8.15(10 \mathrm{H}, \mathrm{m}, \mathrm{Ar}-\mathrm{H}), 10.5(1 \mathrm{H}, \mathrm{s}, \mathrm{COOH})$. Anal. calcd. for $\mathrm{C}_{18} \mathrm{H}_{15} \mathrm{ClN}_{2} \mathrm{O}_{2}$ : C, 66.16; H, 4.63; N, 8.57. Found; C, 66.24; $\mathrm{H}, 4.64 ; \mathrm{N}, 8.53 \%$.

2-((5-Chloro-1,3-diphenyl-1H-pyrazol-4-yl)methyl)propanoic acid (14b): Color: Pale yellow solid. Yield: 86\%. M.p.: $94{ }^{\circ} \mathrm{C}$ (ethanol). IR (KBr, v, cm-1): $3320(\mathrm{OH})$ (br, acid), $3030(\mathrm{CH})$ (Aromatic), 2975 (CH) (alkyl), 1715 (C=0) (acid), 1600, 1580, 1450 (C=C) (Aromatic), 1350, 1140, 755. ${ }^{1} \mathrm{H}$ NMR (90 MHz, $\mathrm{CDCl}_{3}, \delta, \mathrm{ppm}$ ): $1.10\left(\mathrm{~d}, 3 \mathrm{H}, J=10.4 \mathrm{~Hz}, \mathrm{CH}_{3}\right), 2.63(\mathrm{t}, \mathrm{Ha}, J=16.3$ $\mathrm{Hz}, \mathrm{CH}_{2}$ ), 2.73 (ddq, $1 \mathrm{H}, J=9.3 \mathrm{~Hz},-\mathrm{CH}$ ), 2.86 (dd, $\mathrm{Hb}, J=16.3$ $\mathrm{Hz}, \mathrm{CH}_{2}$ ), 6.90-8.25 (m, $\left.10 \mathrm{H}, \mathrm{Ar}-\mathrm{H}\right), 10.8$ (s, 1H, COOH). Anal. calcd. for $\mathrm{C}_{19} \mathrm{H}_{17} \mathrm{ClN}_{2} \mathrm{O}_{2}$ : C, 66.96; H, 5.03; N, 8.22. Found; C, $66.95 ; \mathrm{H}, 4.85 ; \mathrm{N}, 8.37 \%$.

\subsubsection{General procedure for the synthesis of ester 7 or 11a,b or $15 a, b$}

A mixture of acid $\mathbf{6}$ or $\mathbf{1 0 a}, \mathbf{b}$ or $\mathbf{1 4 a}, \mathbf{b}(15 \mathrm{mmol})$, absolute ethanol $(30 \mathrm{~mL})$ and concentrated sulfuric acid $(4 \mathrm{~mL})$ was refluxed for $8 \mathrm{~h}$. The excess alcohol was removed in vacuo and the resulting residue was poured into water $(100 \mathrm{~mL})$, basified by addition of solid $\mathrm{Na}_{2} \mathrm{CO}_{3}$ and left to stand for $3 \mathrm{~h}$ at refrigerator. The product was filtered, washed with water and dried to yield the crude esters. These products were subjected to flash column chromatography (basic alumina, EtOAc $/ n$ hexane, 2/1) to give the pure esters 7 or $\mathbf{1 1 a , b}$ or $\mathbf{1 5 a , b}$ (Scheme 2). Yields, further purifications and spectral data are given in the following:

Ethyl 5-chloro-1,3-diphenyl-1H-pyrazole-4-carboxylate (7): Color: Pale yellow crystals. Yield: $90 \%$. M.p.: $105^{\circ} \mathrm{C}$ (benzene). IR (KBr, v, cm-1): 3070 (CH) (Aromatic), 2965 (CH) (alkyl),
$1715(\mathrm{C}=0)$ (ester), 1600, 1585, 1490, $1440(\mathrm{C}=\mathrm{C})$ (Aromatic), 1340, 1230, 750. ${ }^{1} \mathrm{H}$ NMR $\left(90 \mathrm{MHz}, \mathrm{CDCl}_{3}, \delta, \mathrm{ppm}\right): 1.27(\mathrm{t}, 3 \mathrm{H}, J$ $\left.=7.5 \mathrm{~Hz}, \mathrm{CH}_{3}\right), 4.40\left(\mathrm{q}, 2 \mathrm{H}, J=7.5 \mathrm{~Hz}, \mathrm{CH}_{2}\right), 7.40-8.60(\mathrm{~m}, 10 \mathrm{H}$, Ar-H). Anal. calcd. for $\mathrm{C}_{18} \mathrm{H}_{15} \mathrm{ClN}_{2} \mathrm{O}_{2}: \mathrm{C}, 66.16 ; \mathrm{H}, 4.63 ; \mathrm{N}, 8.57$. Found; C, 66.27; H, 4.36; N, 8.56\%.

Ethyl 2-(4,5-dihydro-5-oxo-1,3-diphenyl-1H-pyrazol-4-yl) acetate (11a): Color: White plates. Yield: 88 \%. M.p.: $142{ }^{\circ} \mathrm{C}$ (ethanol). IR (KBr, v, cm-1): 3083 (CH) (Aromatic), $2980(\mathrm{CH})$ (alkyl), $1745(\mathrm{C}=0)$ (ester), $1682(\mathrm{C}=0)$ (amide), 1605, 1585, 1460, 1455 (C=C) (Aromatic), 1330, 1275, 745. ${ }^{1} \mathrm{H}$ NMR $(90$ $\left.\mathrm{MHz}, \mathrm{CDCl}_{3}, \delta, \mathrm{ppm}\right): 1.32\left(\mathrm{t}, 3 \mathrm{H}, J=7.5 \mathrm{~Hz}, \mathrm{CH}_{3}\right), 2.62(\mathrm{dd}, 1 \mathrm{Ha}, J$ $\left.=14.2 \mathrm{~Hz}, \mathrm{CH}_{2}\right), 2.74\left(\mathrm{t}, 1 \mathrm{Hb}, J=14.2 \mathrm{~Hz}, \mathrm{CH}_{2}\right), 3.27(\mathrm{t}, 1 \mathrm{H}, J=$ $10.4 \mathrm{~Hz}, \mathrm{CH}), 4.22\left(\mathrm{q}, 2 \mathrm{H}, J=7.5 \mathrm{~Hz}, \mathrm{CH}_{2}\right), 7.50-8.30(\mathrm{~m}, 10 \mathrm{H}$, Ar-H). Anal. calcd. for $\mathrm{C}_{19} \mathrm{H}_{18} \mathrm{~N}_{2} \mathrm{O}_{3}$ : C, 70.79; $\mathrm{H}, 5.63 ; \mathrm{N}, 8.69$. Found; C, 70.83; H, 5.74; N, 8.49\%.

Ethyl 2-(4,5-dihydro-5-oxo-1,3-diphenyl-1H-pyrazol-4-yl) propanoate (11b): Color: Reddish viscous oil. Yield: $89 \% \cdot{ }^{\mathrm{n}}{ }^{25}=$ 1.644. IR (Film, v, $\mathrm{cm}^{-1}$ ): $3075(\mathrm{CH})$ (Aromatic), $2977(\mathrm{CH})$ (alkyl), $1740(\mathrm{C}=0)$ (ester), $1673(\mathrm{C}=0)$ (amide), 1600, 1585, 1495, 1455 (C=C) (Aromatic), 1340, 1245, 745. ${ }^{1} \mathrm{H}$ NMR (90 $\left.\mathrm{MHz}, \mathrm{CDCl}_{3}, \delta, \mathrm{ppm}\right): 1.15\left(\mathrm{~d}, 3 \mathrm{H}, J=9.4 \mathrm{~Hz}, \mathrm{CH}_{3}\right), 1.35(\mathrm{t}, 3 \mathrm{H}, J=$ $\left.7.5 \mathrm{~Hz}, \mathrm{CH}_{3}\right), 2.82(\mathrm{p}, 1 \mathrm{H}, J=12.5 \mathrm{~Hz}, \mathrm{CH}), 3.24$ (app. t, $1 \mathrm{H}, J=$ $12.5 \mathrm{~Hz}, \mathrm{CH}), 4.20\left(\mathrm{q}, 2 \mathrm{H}, J=7.5 \mathrm{~Hz}, \mathrm{CH}_{2}\right), 7.45-8.50(\mathrm{~m}, 10 \mathrm{H}$, Ar-H). Anal. calcd. for $\mathrm{C}_{20} \mathrm{H}_{20} \mathrm{~N}_{2} \mathrm{O}_{3}$ : C, 71.41; H, 5.99; N, 8.33. Found; C, 71.44; H, 6.14; N, 8.09\%.

Ethyl 3-(5-chloro-1,3-diphenyl-1H-pyrazol-4-yl)propanoate (15a): Color: Yellowish viscous oil. Yield: $85 \% .{ }^{n^{2}}{ }^{25}=1.584$. IR (Film, v, $\mathrm{cm}^{-1}$ ): $3055(\mathrm{CH})$ (Aromatic), $2964(\mathrm{CH})$ (alkyl), 1738 $(\mathrm{C}=0)$ (ester), 1590, 1550, 1460, $1430(\mathrm{C}=\mathrm{C})$ (Aromatic), 1335, 1265, 750. ${ }^{1} \mathrm{H}$ NMR $\left(90 \mathrm{MHz}, \mathrm{CDCl}_{3}, \delta, \mathrm{ppm}\right): 1.30(\mathrm{t}, 3 \mathrm{H}, J=7.5$ $\left.\mathrm{Hz}, \mathrm{CH}_{3}\right), 2.52\left(\mathrm{t}, 2 \mathrm{H}, J=6.2 \mathrm{~Hz}, \mathrm{CH}_{2}\right), 2.95\left(\mathrm{t}, 2 \mathrm{H}, J=6.2 \mathrm{~Hz}, \mathrm{CH}_{2}\right)$, $4.10\left(\mathrm{q}, 2 \mathrm{H}, J=7.5 \mathrm{~Hz}, \mathrm{CH}_{2}\right), 7.22-8.45(\mathrm{~m}, 10 \mathrm{H}, \mathrm{Ar}-\mathrm{H})$. Anal. calcd. for $\mathrm{C}_{20} \mathrm{H}_{19} \mathrm{ClN}_{2} \mathrm{O}_{2}: \mathrm{C}, 67.70 ; \mathrm{H}, 5.40 ; \mathrm{N}, 7.89$. Found; C, 67.91; $\mathrm{H}, 5.37 ; \mathrm{N}, 7.90 \%$.

Ethyl 2-((5-chloro-1,3-diphenyl-1H-pyrazol-4-yl)methyl) propanoate (15b): Color: Pale yellow crystals. Yield: $86 \%$. M.p.: $74{ }^{\circ} \mathrm{C}$ (ethanol). IR (KBr, v, cm${ }^{-1}$ ): $3105(\mathrm{CH})$ (Aromatic), 2985 (CH) (alkyl), 1742 (C=0) (ester), 1600, 1560, 1485, $1440(\mathrm{C}=\mathrm{C})$ (Aromatic), 1370, 1220, 747. ${ }^{1} \mathrm{H}$ NMR $\left(90 \mathrm{MHz}, \mathrm{CDCl}_{3}, \delta, \mathrm{ppm}\right)$ : 1.12 (d, 3H, $J=10.4 \mathrm{~Hz}, \mathrm{CH}_{3}$ ), 1.25 (t, $3 \mathrm{H}, J=7.5 \mathrm{~Hz}, \mathrm{CH}_{3}$ ), 2.66 (q, Ha, $J=16.3 \mathrm{~Hz}, \mathrm{CH}_{2}$ ), 2.71 (ddq, $1 \mathrm{H}, J=9.3 \mathrm{~Hz},-\mathrm{CH}$ ), 2.94 (dd, $\mathrm{Hb}, J=16.3 \mathrm{~Hz}, \mathrm{CH}_{2}$ ), 4.25 (q, $\left.2 \mathrm{H}, J=7.5 \mathrm{~Hz}, \mathrm{CH}_{2}\right), 6.95-8.25$ (m, $10 \mathrm{H}, \mathrm{Ar}-\mathrm{H}$ ). Anal. calcd. for $\mathrm{C}_{21} \mathrm{H}_{21} \mathrm{ClN}_{2} \mathrm{O}_{2}: \mathrm{C}, 68.38 ; \mathrm{H}, 5.74 ; \mathrm{N}$, 7.59. Found; C, 68.36; H, 5.69; N, 7.65\%. 
Table 1. Optimum conditions for the synthesis of heteroarylalkanols 8, 12a,b and 16a,b.

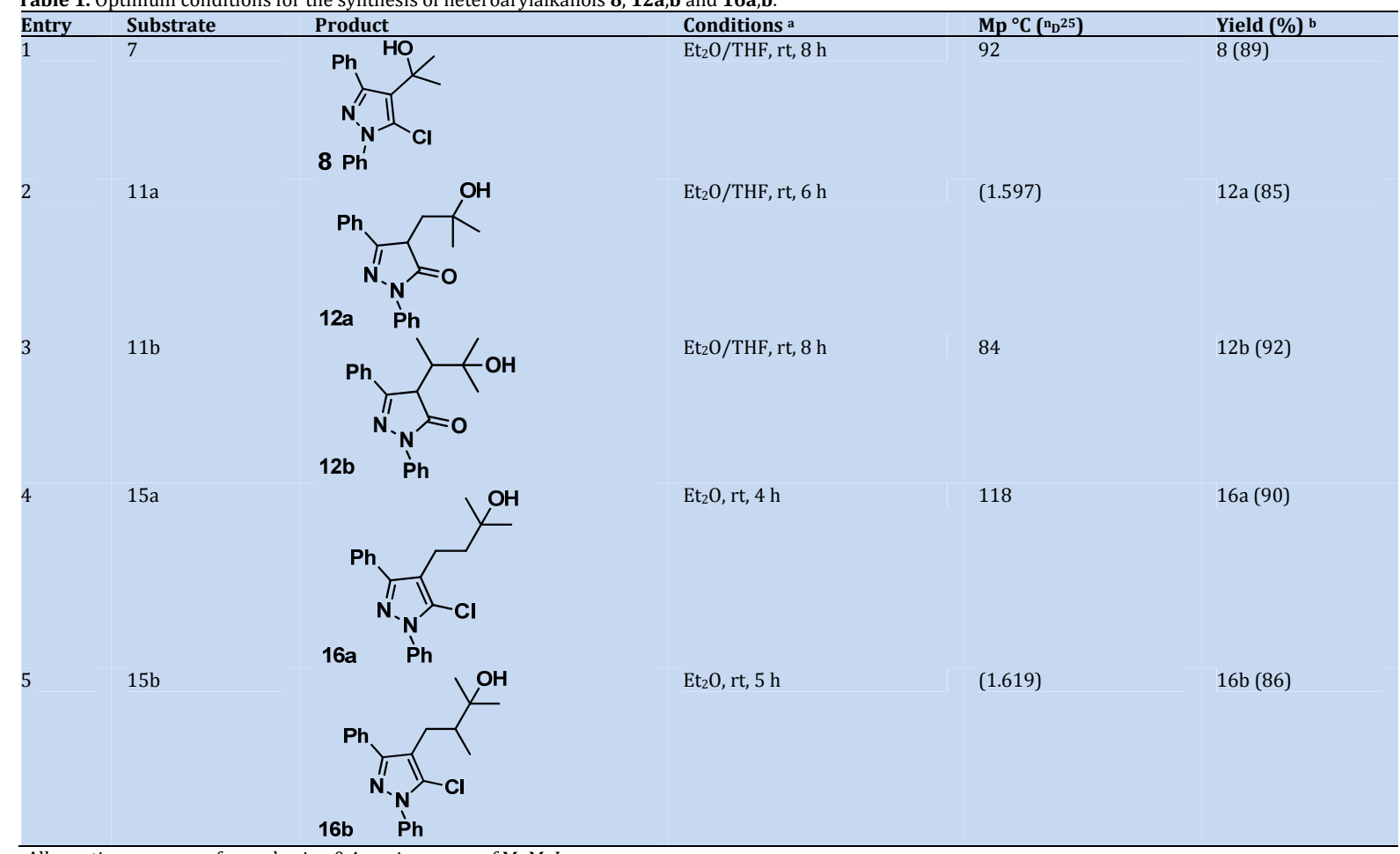

a All reactions were performed using 0.4 equiv. excess of MeMgI.

b Isolated yield refer to substrate.

\subsubsection{General procedure for the synthesis of alcohols 8 , $12 a, b, 16 a, b$}

To an ice-cold Grignard reagent solution obtained as usual from $\mathrm{Mg}$ turnings $(0.32 \mathrm{~g}, 13 \mathrm{mmol})$, alkyl or aryl halide (13 $\mathrm{mmol})$ in ether $(40 \mathrm{~mL})$, was added a solution of esters 7 or $\mathbf{1 1 a}, \mathbf{b}$ or $\mathbf{1 5 a}, \mathbf{b}(4.6 \mathrm{mmol})$ in ether or THF $(30 \mathrm{~mL})$. The reaction mixture was stirred at required temperature for appointed time (Table 1) followed by decomposition with saturated aqueous $\mathrm{NH}_{4} \mathrm{Cl}$ solution. The product was extracted with ether $(3 \times 30 \mathrm{~mL})$ and the combined organic phases were washed with water, dried over anhydrous $\mathrm{MgSO}_{4}$. The solvent was removed in vacuo and the residue was purified by flash column chromatography (basic alumina, EtOAc: $n$-hexane, 3:1, $v: v)$ resulting pure alcohols 8 or $\mathbf{1 2 a}, \mathbf{b}$ or $\mathbf{1 6} \mathbf{a}, \mathbf{b}$ (Scheme 2). The conditions and isolated yields are shown in Table 1 and spectral data are given in the following:

2-(5-Chloro-1,3-diphenyl-1H-pyrazol-4-yl)propan-2-ol (8): Color: White plates. Yield: $80 \%$. M.p.: $92{ }^{\circ} \mathrm{C}$ (cyclohexane). IR (KBr, v, cm-1): $3340(\mathrm{OH})$ (alcohol), $3060(\mathrm{CH})$ (Aromatic), 2975 (CH) (alkyl), 1610, 1560, 1450, 1445 (C=C) (Aromatic), 1375, 1330, 760. ${ }^{1} \mathrm{H}$ NMR $\left(400 \mathrm{MHz}, \mathrm{CDCl}_{3}, \delta, \mathrm{ppm}\right): 1.40(\mathrm{~s}, 6 \mathrm{H}$, $\left.2 \mathrm{CH}_{3}\right), 3.5\left(\mathrm{~s}, 1 \mathrm{H}, \mathrm{OH}\right.$ exchangeable with $\left.\mathrm{D}_{2} \mathrm{O}\right)$ 7.50-8.60 (m, $10 \mathrm{H}, \mathrm{Ar}-\mathrm{H})$. MS (EI, $m / z(\%)): 314\left(\mathrm{M}^{+}+2,6\right), 312\left(\mathrm{M}^{+}, 17\right), 310$ (40), 294 (100), 277 (61), 253 (82), 235 (52), 194 (42), 180 (35), 177 (18), 168 (14), 158 (37), 103 (33), 91 (27), 77 (27). Anal. calcd. for $\mathrm{C}_{18} \mathrm{H}_{17} \mathrm{ClN}_{2} \mathrm{O}: \mathrm{C}, 69.12 ; \mathrm{H}, 5.48 ; \mathrm{N}, 8.96$. Found; $\mathrm{C}$, 69.31; H, 5.26; N, 8.82\%.

4-(2-Hydroxy-2-methylpropyl)-1,3-diphenyl-1H-pyrazol-5 (4H)-one (12a): Color: Pale yellow viscous oil. Yield: $81 \%$. ${ }^{\mathrm{n}} 25$ $=1.597$. IR (Film, v, cm$\left.{ }^{-1}\right): 3440(\mathrm{OH})$ (alcohol), $3035(\mathrm{CH})$ (Aromatic), $2970(\mathrm{CH})$ (alkyl), 1665 (C=0) (amide), 1600, 1580, 1455, 1445 (C=C) (Aromatic), 1390, 1330, 745. ${ }^{1} \mathrm{H}$ NMR (400 $\mathrm{MHz}, \mathrm{CDCl}_{3}, \delta, \mathrm{ppm}$ ): 1.30 (s, $6 \mathrm{H}, 2 \mathrm{CH}_{3}$ ), 1.74 (app. t, $2 \mathrm{H}, J=14.2$ $\left.\mathrm{Hz}, \mathrm{CH}_{2}\right), 2.55\left(\mathrm{~s}, 1 \mathrm{H}, \mathrm{OH}\right.$ exchangeable with $\left.\mathrm{D}_{2} \mathrm{O}\right), 2.65(\mathrm{t}, 1 \mathrm{H}, J=$ $14.2 \mathrm{~Hz}, \mathrm{CH}$ ), 7.25-8.22 (m, 10H, Ar-H). MS (EI, $m / z$ (\%)): 308
$\left(\mathrm{M}^{+}, 13\right), 290$ (100), 249 (55), 231 (41), 235 (55), 193 (20), 180 (29), 178 (15), 167 (11), 158 (35), 103 (24), 90 (22), 77 (44). Anal. calcd. for $\mathrm{C}_{19} \mathrm{H}_{20} \mathrm{~N}_{2} \mathrm{O}_{2}$ : C, 74.00; H, 6.54; N, 9.08. Found; C, 74.18; $\mathrm{H}, 6.40 ; \mathrm{N}, 9.10 \%$.

4-(3-Hydroxy-3-methylbutan-2-yl)-1,3-diphenyl-1H-pyrazol5(4H)-one (12b): Color: Cream plates. Yield: $84 \%$. M.p.: $84{ }^{\circ} \mathrm{C}$ (benzene). IR (KBr, $\left.v, \mathrm{~cm}^{-1}\right): 3425(\mathrm{OH})$ (br, alcohol), $3060(\mathrm{CH})$ (Aromatic), 2965 (CH) (alkyl), 1668 (C=0) (amide), 1594, 1580, 1455, 1440 (C=C) (Aromatic), 1385, 1320, 743. ${ }^{1} \mathrm{H}$ NMR (400 $\mathrm{MHz}, \mathrm{CDCl}_{3}, \delta, \mathrm{ppm}$ ): 0.90 (d, 3H, $\left.J=9.4 \mathrm{~Hz}, \mathrm{CH}_{3}\right), 1.27(\mathrm{~s}, 6 \mathrm{H}$, $\left.2 \mathrm{CH}_{3}\right), 1.85(\mathrm{p}, 1 \mathrm{H}, J=12.5 \mathrm{~Hz},-\mathrm{CH}), 2.10(\mathrm{~s}, 1 \mathrm{H}, \mathrm{OH}$ exchangeable with $\left.\mathrm{D}_{2} \mathrm{O}\right), 2.42(\mathrm{~d}, 1 \mathrm{H}, J=12.5 \mathrm{~Hz}, \mathrm{CH}), 7.15-8.25$ (m, 10H, Ar-H). MS (EI, $m / z(\%)): 322\left(\mathrm{M}^{+}, 10\right), 304(100), 263$ (31), 245 (75), 248 (62), 235 (33), 194 (37), 180 (20), 177 (14), 168 (21), 158 (48), 103 (23), 91 (35), 77 (47). Anal. calcd. for $\mathrm{C}_{20} \mathrm{H}_{22} \mathrm{~N}_{2} \mathrm{O}_{2}$ : C, 74.51; $\mathrm{H}, 6.88 ; \mathrm{N}, 8.69$. Found; $\mathrm{C}, 74.62 ; \mathrm{H}, 6.69$; N, $8.63 \%$.

4-(5-Chloro-1,3-diphenyl-1H-pyrazol-4-yl)-2-methylbutan-2ol (16a): Color: White needles. Yield: 85 \%. M.p.: $118{ }^{\circ} \mathrm{C}$ (benzene). IR (KBr, v, cm-1): $3490(\mathrm{OH})$ (alcohol), $3075(\mathrm{CH})$ (Aromatic), 2990 (CH) (alkyl), 1610, 1490, 1455, 1430 (C=C) (Aromatic), 1386, 1355, 1020, 745. ${ }^{1} \mathrm{H}$ NMR $\left(400 \mathrm{MHz}, \mathrm{CDCl}_{3}, \delta\right.$, ppm): $1.10\left(\mathrm{~s}, 6 \mathrm{H}, 2 \mathrm{CH}_{3}\right), 1.55\left(\mathrm{t}, 2 \mathrm{H}, J=6.2 \mathrm{~Hz}, \mathrm{CH}_{2}\right), 1.80(\mathrm{~s}$, $1 \mathrm{H}, \mathrm{OH}$ exchangeable with $\left.\mathrm{D}_{2} \mathrm{O}\right), 2.40\left(\mathrm{t}, 2 \mathrm{H}, J=6.2 \mathrm{~Hz}, \mathrm{CH}_{2}\right)$, 7.22-8.30 (m, $10 \mathrm{H}, \mathrm{Ar}-\mathrm{H})$. MS (EI, $m / z(\%)): 342\left(\mathrm{M}^{+}+2,9\right), 340$ $\left(\mathrm{M}^{+}, 22\right), 338$ (48), 322 (100), 281 (51), 267 (42), 263 (12), 253 (70), 245 (38), 235 (18), 194 (42), 180 (11), 176 (19), 167 (30), 159 (43), 103 (13), 91 (38), 77 (39). Anal. calcd. for $\mathrm{C}_{20} \mathrm{H}_{21} \mathrm{ClN}_{2} \mathrm{O}$ : C, 70.48; $\mathrm{H}, 6.21 ; \mathrm{N}, 8.22$. Found; C, 70.49; $\mathrm{H}, 6.26$; $\mathrm{N}, 8.05 \%$.

4-(5-Chloro-1,3-diphenyl-1H-pyrazol-4-yl)-2,3-dimethyl butan-2-ol (16b): Color: Reddish viscous oil. Yield: $81 \%$. ${ }^{\mathrm{n}_{\mathrm{D}}}{ }^{25}=$ 1.619. IR (Film, $v, \mathrm{~cm}^{-1}$ ): $3465(\mathrm{OH})$ (alcohol), $3055(\mathrm{CH})$ (Aromatic), 2973 (CH) (alkyl), 1590, 1480, 1455, 1440 (C=C) (Aromatic), 1345, 745. 
Table 2. Friedel-Crafts ring closures of heterocyclic alkanols $8,12 \mathbf{a}, \mathbf{b}$ and $\mathbf{1 6} \mathbf{a}, \mathbf{b}$

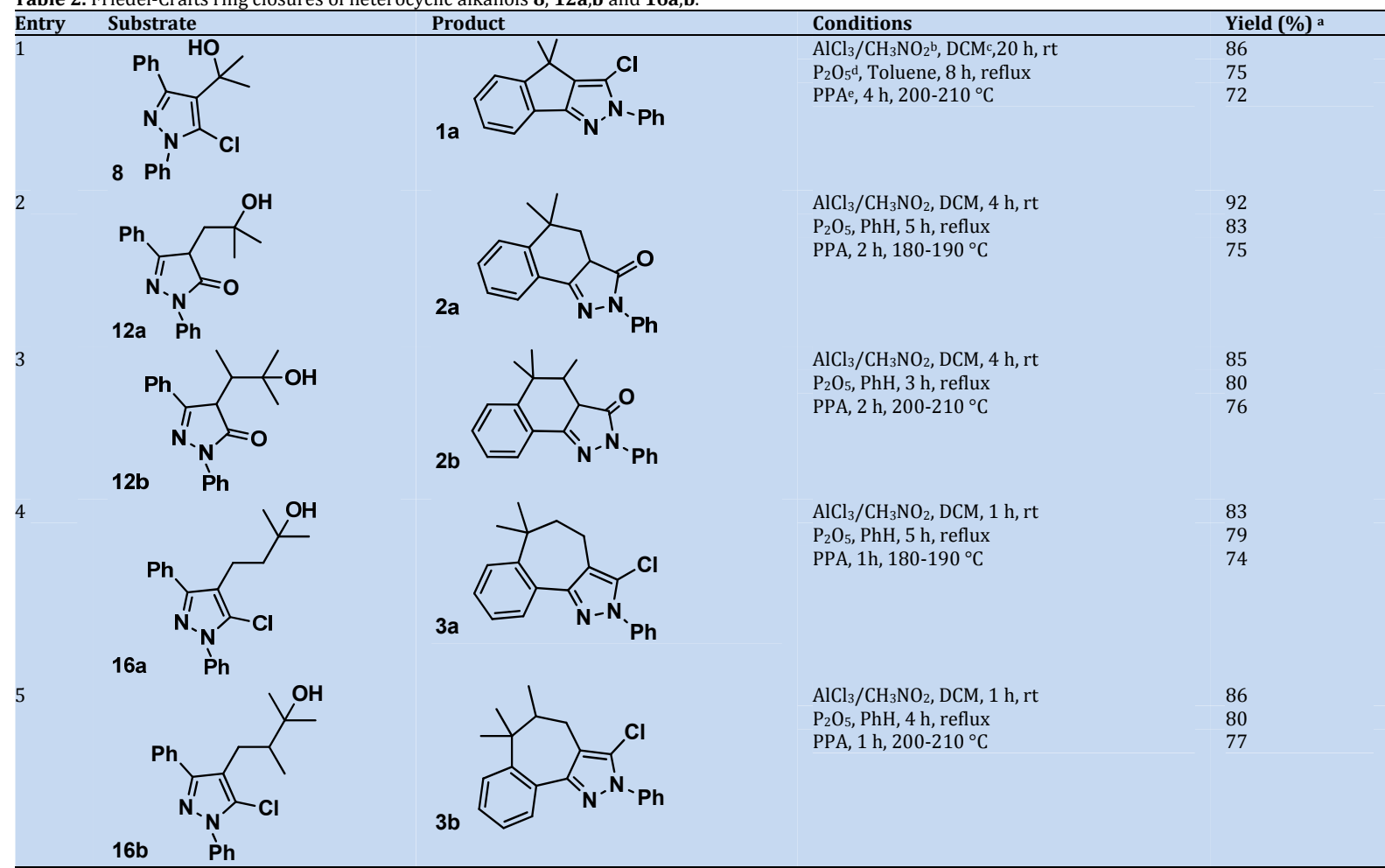

a Isolated yield refer to substrate.

b With $\mathrm{AlCl}_{3} / \mathrm{CH}_{3} \mathrm{NO}_{2}$ catalyst reactant proportions were: carbinol or acid (0.002 moe), $\mathrm{AlCl}_{3}(0.0024 \mathrm{~mol}), \mathrm{CH}_{3} \mathrm{NO}_{2}(0.024 \mathrm{~mol})$, solvent $(10 \mathrm{~mL})$. c Dichloromethane.

${ }^{d}$ With $\mathrm{P}_{2} \mathrm{O}_{5}$ catalyst reactant proportions were: carbinol or acid $(0.4 \mathrm{~g})$ and $\mathrm{P}_{2} \mathrm{O}_{5}(4 \mathrm{~g})$ in anhydrous toluene or benzene $(15 \mathrm{~mL})$.

e With PPA catalyst reactant proportions were: carbinol or acid ( $0.5 \mathrm{~g})$ and PPA (5 g).

${ }^{1} \mathrm{H}$ NMR $\left(400 \mathrm{MHz}, \mathrm{CDCl}_{3}, \delta, \mathrm{ppm}\right): 0.95(\mathrm{~d}, 3 \mathrm{H}, J=10.4 \mathrm{~Hz}$, $\left.\mathrm{CH}_{3}\right), 1.30\left(\mathrm{~s}, 6 \mathrm{H}, 2 \mathrm{CH}_{3}\right), 2,21(\mathrm{dt}, 1 \mathrm{H}, J=9.3 \mathrm{~Hz},-\mathrm{CH}), 2.42(\mathrm{t}$, $\mathrm{Ha}, J=16.3 \mathrm{~Hz}, \mathrm{CH}_{2}$ ), 2.64 (app.t, $\mathrm{Hb}, J=16.3 \mathrm{~Hz}, \mathrm{CH}_{2}$ ), 2.50 (s, $1 \mathrm{H}, \mathrm{OH}$ exchangeable with $\left.\mathrm{D}_{2} \mathrm{O}\right), 7.22-8.15(\mathrm{~m}, 10 \mathrm{H}, \mathrm{Ar}-\mathrm{H})$. MS (EI, $m / z(\%)): 356\left(\mathrm{M}^{+}+2,5\right), 354\left(\mathrm{M}^{+}, 13\right), 336(100), 295$ (74), 280 (48), 267 (35), 252 (84), 245 (24), 235 (13), 194 (48), 180 (14), 176 (26), 167 (35), 159 (52), 103 (15), 91 (48), 77 (42). Anal. calcd. for $\mathrm{C}_{21} \mathrm{H}_{23} \mathrm{ClN}_{2} \mathrm{O}: \mathrm{C}, 71.07 ; \mathrm{H}, 6.53 ; \mathrm{N}, 7.89$. Found; C, 71.11; $\mathrm{H}, 6.53 ; \mathrm{N}, 7.65 \%$.

\subsubsection{Friedel-Crafts cyclialkylations procedures}

The procedures [46-54] described earlier for cyclialkylation of acids and alcohols with $\mathrm{AlCl}_{3} / \mathrm{CH}_{3} \mathrm{NO}_{2}$ or $\mathrm{P}_{2} \mathrm{O}_{5}$ or PPA catalysts were essentially followed. In all reactions, the crude oily or solid products were purified by flash column chromatography (basic alumina, EtOAc: $n$-hexane, $3: 1, v: v$ ) gave the pure products $1 \mathbf{a}, \mathbf{b}, \mathbf{2 a - d}$ and $3 \mathbf{3}-\mathbf{d}$. The conditions and yields for all products are shown in Table 2 and 3 while the physical and spectral data of the products are given below.

3-Chloro-2,4-dihydro-4,4-dimethyl-2-phenylindeno[1,2-c] pyrazole (1a): Color: White needles. M.p.: $117^{\circ} \mathrm{C}$ (acetone). IR (KBr, v, cm-1): 3075 (CH) (Aromatic), 2980 (CH) (alkyl), 1600, 1480, 1460, 1435 (C=C) (Aromatic), 1355, 1220, 748. ${ }^{1} \mathrm{H}$ NMR $\left(400 \mathrm{MHz}, \mathrm{CDCl}_{3}, \delta, \mathrm{ppm}\right): 1.42\left(\mathrm{~s}, 6 \mathrm{H}, 2 \mathrm{CH}_{3}\right), 7.15-8.35(\mathrm{~m}, 9 \mathrm{H}$, Ar-H). ${ }^{13} \mathrm{C}$ NMR $\left(100 \mathrm{MHz}, \mathrm{CDCl}_{3}, \delta, \mathrm{ppm}\right): 27.6\left(1 \mathrm{C},-\mathrm{C}\left(\mathrm{CH}_{3}\right)_{2}\right)$, $31.4\left(2 \mathrm{C},-\mathrm{C}\left(\mathrm{CH}_{3}\right)_{2}\right), 117.4$ (1C, $\left.\mathrm{C}-3 \mathrm{a}\right), 123.4$ (2C, Ar., C-2', C-6'), 126.3 (1C, Ar., C-4'), 126.7 (1C, Ar., C-7), 127.4 (1C, Ar., C-5), $128.2(1 \mathrm{C}, \mathrm{N}-\mathrm{C}(\mathrm{Cl})=, \mathrm{C}-3), 129.2(1 \mathrm{C}, \mathrm{Ar} ., \mathrm{C}-8), 129.4$ (1C, Ar., C6), 132.4 (2C, Ar., C-3', C-5'), 134.7 (1C, Ar., C-8a), 142.7 (1C, Ar., C-1'), 156.1 (1C, N=C-, C-8b), 160.4 (1C, Ar., C-4a). Anal. calcd. for $\mathrm{C}_{18} \mathrm{H}_{15} \mathrm{ClN}_{2}$ : C, 73.34; $\mathrm{H}, 5.13 ; \mathrm{N}$, 9.50. Found; C, 73.38; $\mathrm{H}, 5.14 ; \mathrm{N}, 9.37 \%$.
4,5-Dihydro-5,5-dimethyl-2-phenyl-2H-benzo[g]indazol-3 (3aH)-one (2a): Color: White needles. M.p.: $92{ }^{\circ} \mathrm{C}$ (acetone). IR (KBr, v, cm $\left.{ }^{-1}\right): 3090(\mathrm{CH})$ (Aromatic), $2980(\mathrm{CH})$ (alkyl), 1674 $(\mathrm{C}=0)$ (amide), 1600, 1475, 1465, 1440 (C=C) (Aromatic), 1350, 750. ${ }^{1} \mathrm{H}$ NMR $\left(400 \mathrm{MHz}, \mathrm{CDCl}_{3}, \delta, \mathrm{ppm}\right): 1.40\left(\mathrm{~s}, 6 \mathrm{H}, 2 \mathrm{CH}_{3}\right), 1.84$ (t, $\left.1 \mathrm{Ha}, J=14.2 \mathrm{~Hz}, \mathrm{CH}_{2}\right), 2.17$ (dd, $1 \mathrm{Hb}, J=14.2 \mathrm{~Hz}, \mathrm{CH}_{2}$ ), 2.35 (app. q, $1 \mathrm{H}, J=10.4 \mathrm{~Hz}, \mathrm{CH}), 6.85-8.11(9 \mathrm{H}, \mathrm{m}, \mathrm{Ar}-\mathrm{H}) .{ }^{13} \mathrm{C}$ NMR $\left(100 \mathrm{MHz}, \mathrm{CDCl}_{3}, \delta, \mathrm{ppm}\right): 29.2\left(2 \mathrm{C},-\mathrm{C}\left(\mathrm{CH}_{3}\right)_{2}\right), 33.4(1 \mathrm{C}$, $\left.\mathrm{C}\left(\mathrm{CH}_{3}\right)_{2}\right), 43.6\left(1 \mathrm{C},-\mathrm{CH}_{2-}, \mathrm{C}-4\right), 44.3$ (1C, -CH-CO-, C-3a), 122.5 (2C, Ar., C-2', C-6'), 124.4 (1C, Ar., C-4'), 125.2 (1C, Ar., C-6), 126.4 (1C, Ar., C-9a), 127.6 (1C, Ar., C-8), 128.8 (1C, Ar., C-9), 131.4 (2C, Ar., C-3', C-5'), 133.7 (1C, Ar., C-7), 144.4 (1C, Ar., C1'), 153.7 (1C, N=C-, C-9b), 157.5 (1C, Ar., C-5a), 178.2 (1C, C=0, C-3). Anal. calcd. for $\mathrm{C}_{19} \mathrm{H}_{18} \mathrm{~N}_{2} \mathrm{O}$ : C, 78.59; H, 6.25; N, 9.65 . Found; C, 78.55; H, 6.37; N, 9.49\%.

4,5-Dihydro-4,5,5-trimethyl-2-phenyl-2H-benzo[g]indazol3(3aH)-one (2b): Color: Reddish viscous oil. $\mathrm{n}_{\mathrm{D}}^{25}=1.592$. IR (Film, v, cm-1): 3062 (CH) (Aromatic), 2955 (CH) (alkyl), 1665 (C=0) (amide), 1590, 1490, 1470, 1445 (C=C) (Aromatic), 1345, 7453. ${ }^{1 \mathrm{H}} \mathrm{NMR}\left(400 \mathrm{MHz}, \mathrm{CDCl}_{3}, \delta, \mathrm{ppm}\right): 1.10(\mathrm{~d}, 3 \mathrm{H}, J=9.4 \mathrm{~Hz}$, $\left.\mathrm{CH}_{3}\right), 1.37\left(\mathrm{~s}, 6 \mathrm{H}, 2 \mathrm{CH}_{3}\right), 2.21(\mathrm{p}, 1 \mathrm{H}, J=12.5 \mathrm{~Hz}, \mathrm{CH}$ ), 2.38 (app. t, $1 \mathrm{H}, J=12.5 \mathrm{~Hz}, \mathrm{CH}), 7.19-8.35(\mathrm{~m}, 9 \mathrm{H}, \mathrm{Ar}-\mathrm{H}) .{ }^{13} \mathrm{C}$ NMR $(100$ $\left.\mathrm{MHz}, \mathrm{CDCl}_{3}, \delta, \mathrm{ppm}\right): 14.2\left(1 \mathrm{C},-\mathrm{CH}\left(\mathrm{CH}_{3}\right)\right), 28.3\left(2 \mathrm{C},-\mathrm{C}\left(\mathrm{CH}_{3}\right)_{2}\right)$, $37.6\left(1 \mathrm{C},-\mathrm{C}\left(\mathrm{CH}_{3}\right)_{2}, \mathrm{C}-5\right), 42.8\left(1 \mathrm{C},-\mathrm{C}\left(\mathrm{CH}_{3}\right), \mathrm{C}-4\right), 44.2(1 \mathrm{C}$, COCH-, C-3a), 121.8 (2C, Ar., C-2', C-6'), 124.8 (1C, Ar., C-4'), 125.2 (1C, Ar., C-6), 126.7 (1C, Ar., C-9a), 127.2 (1C, Ar., C-8), 128.8 (1C, Ar., C-9), 132.2 (2C, Ar., C-3', C-5'), 133.4 (1C, Ar., C7), 144.2 (1C, Ar., C-1'), 151.7 (1C, N=C-,C-9b), 157.5 (1C, Ar., C5a), 181.3 (1C, $\mathrm{C}=0, \mathrm{C}-3$ ). Anal. calcd. for $\mathrm{C}_{20} \mathrm{H}_{20} \mathrm{~N}_{2} \mathrm{O}$ : C, 78.92; $\mathrm{H}$, 6.62; N, 9.20. Found; C, 78.79; H, 6.44; N, 9.36\%.

3-Chloro-6,6-dimethyl-4,5,6-trihydro-2-phenyl-benzo[6,7] cyclohepta[1,2-c]pyrazole (3a): Color: Reddish viscous oil. ${ }^{n^{25}}=$ 1.589. 
Table 3. Friedel-Crafts ring closures of heterocyclic acids 6 or $10 a, b$ or $14 a, b$.

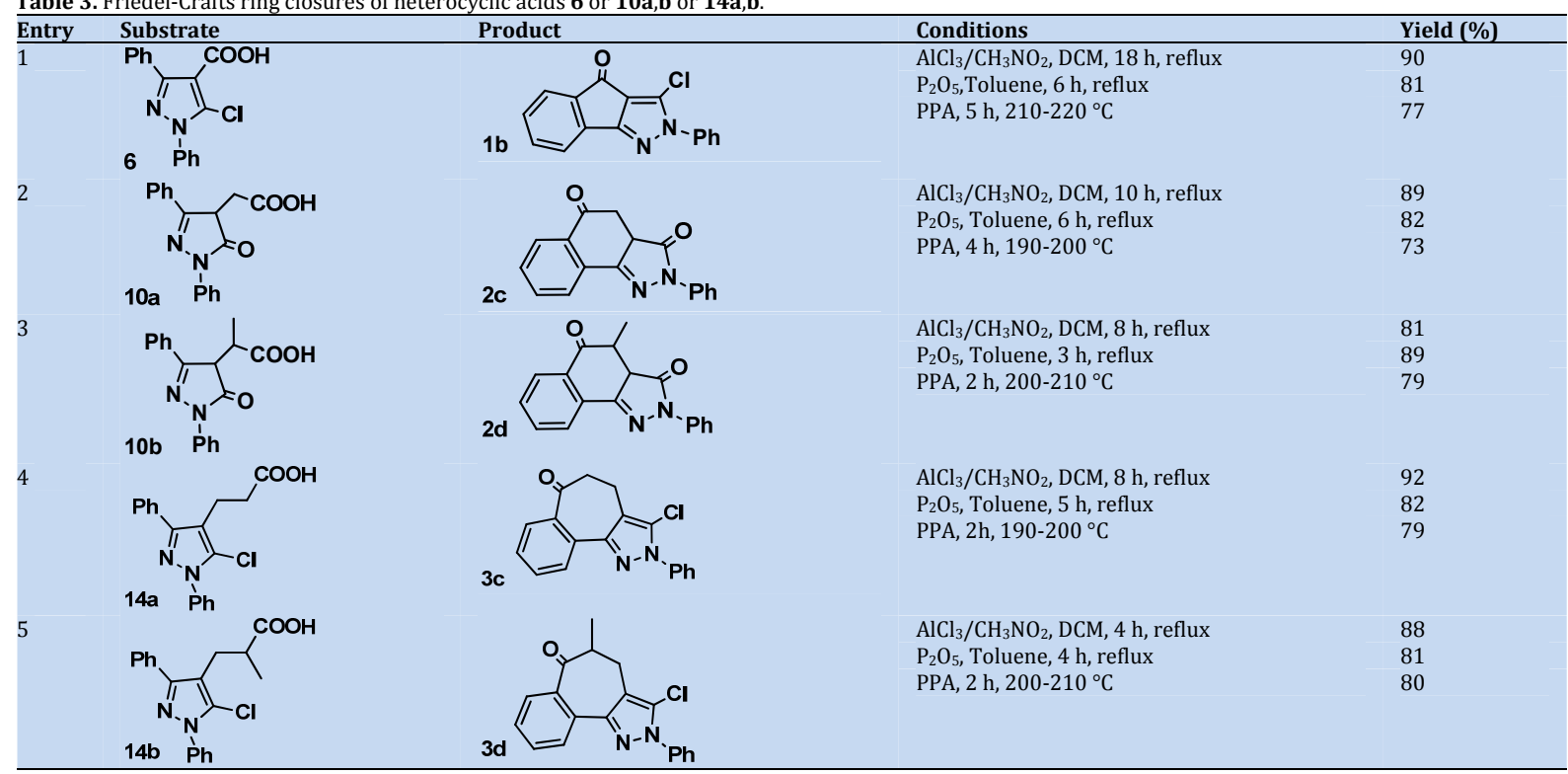

IR (Film, v, $\mathrm{cm}^{-1}$ ): 3090 (CH) (Aromatic), 2980 (CH) (alkyl), 1604, 1485, 1465, 1442 (C=C) (Aromatic), 1350, 695. ${ }^{1} \mathrm{H}$ NMR (400 MHz, $\left.\mathrm{CDCl}_{3}, \delta, \mathrm{ppm}\right): 1.27\left(\mathrm{~s}, 6 \mathrm{H}, 2 \mathrm{CH}_{3}\right), 1.75(\mathrm{t}, 2 \mathrm{H}, J=6.2$ $\left.\mathrm{Hz}, \mathrm{CH}_{2}\right), 2.64\left(\mathrm{t}, 2 \mathrm{H}, J=6.2 \mathrm{~Hz}, \mathrm{CH}_{2}\right), 7.21-8.14(9 \mathrm{H}, \mathrm{m}, \mathrm{Ar}-\mathrm{H})$. ${ }^{13} \mathrm{C}$ NMR $\left(100 \mathrm{MHz}, \mathrm{CDCl}_{3}, \delta, \mathrm{ppm}\right): 17.5\left(1 \mathrm{C},-\mathrm{CH}_{2^{-}}, \mathrm{C}-4\right), 31.5$ (2C, $\left.-\mathrm{C}\left(\mathrm{CH}_{3}\right)_{2}\right), 42.6\left(1 \mathrm{C},-\mathrm{C}\left(\mathrm{CH}_{3}\right)_{2}, \mathrm{C}-6\right), 49.2$ (1C, $\left.-\mathrm{CH}_{2}, \mathrm{C}-5\right)$, 119.3 (1C, C-3a), 122.4 (2C, Ar., C-2', C-6'), 125.6 (1C, Ar., C-7), 125.8 (1C, Ar., C-10a), 126.2 (1C, Ar., C-9), 126.8 (1C, Ar., C-4'), $127.4(1 \mathrm{C}, \mathrm{N}-\mathrm{C}(\mathrm{Cl})=, \mathrm{C}-3), 127.8$ (1C, Ar., C-10), 128.4 (1C, Ar., C8), 131.2 (2C, Ar., C-3', C-5'), 142.7 (1C, Ar., C-1'), 149.1 (1C, Ar., C-6a), 154.5 (1C, N=C-, C-10b). Anal. calcd. for $\mathrm{C}_{20} \mathrm{H}_{19} \mathrm{ClN}_{2}$ : C, 74.41; H, 5.93; N, 8.68. Found; C, 74.23; H, 6.08; N, 8.46\%.

3-Chloro-5,6,6-trimethyl-4,5,6-trihydro-2-phenyl-benzo[6,7] cyclohepta[1,2-c]pyrazole (3b): Color: White needles. M.p.: 83 ${ }^{\circ} \mathrm{C}$ (benzene). IR (KBr, $\left.v, \mathrm{~cm}^{-1}\right): 3055(\mathrm{CH})$ (Aromatic), 2954 $(\mathrm{CH})$ (alkyl), 1600, 1580, 1480, $1465(\mathrm{C}=\mathrm{C})$ (Aromatic), 1345, 1285, 1020, 746. ${ }^{1} \mathrm{H}$ NMR $\left(400 \mathrm{MHz}, \mathrm{CDCl}_{3}, \delta, \mathrm{ppm}\right): 0.92$ (d, 3H, $\left.J=6 \mathrm{~Hz}, \mathrm{CH}_{3}\right), 1.35\left(\mathrm{~s}, 6 \mathrm{H}, 2 \mathrm{CH}_{3}\right), 2.33$ (dd, $\mathrm{Ha}, J=16.3 \mathrm{~Hz}, \mathrm{CH}_{2}$ ), 2.42 (p, $1 \mathrm{H}, J=9.3 \mathrm{~Hz},-\mathrm{CH}$ ), 2.64 (app.t, $\mathrm{Hb}, J=16.3 \mathrm{~Hz}, \mathrm{CH}_{2}$ ), 7.11-8.25 (m, 9H, Ar-H). ${ }^{13} \mathrm{C}$ NMR (100 MHz, $\left.\mathrm{CDCl}_{3}, \delta, \mathrm{ppm}\right):$ $16.4\left(1 \mathrm{C},-\mathrm{CHCH}_{3}\right), 27.3\left(2 \mathrm{C},-\mathrm{C}\left(\mathrm{CH}_{3}\right)_{2}\right), 29.2\left(1 \mathrm{C},-\mathrm{CH}_{2}-\mathrm{C}-4\right), 42.5$ (1C, $\left.-\mathrm{C}\left(\mathrm{CH}_{3}\right)_{2}, \mathrm{C}-6\right), 52.4\left(1 \mathrm{C},-\mathrm{CHCH}_{3}, \mathrm{C}-5\right), 119.2$ (1C, $\left.\mathrm{C}-3 \mathrm{a}\right)$, 120.7 (2C, Ar., C-2', C-6'), 125.2 (1C, Ar., C-7), 125.6 (1C, Ar., C10a), 126.6 (2C, Ar., C-4', C-9), 127.2 (1C, N-C(Cl)=, C-3), 128.1 (1C, Ar., C-10), 128.9 (1C, Ar., C-8), 129.5 (2C, Ar., C-3', C-5'), 142.7 (1C, Ar., C-1'), 151.4 (1C, Ar., C-6a), 157.4 (1C, N=C-,C10b). Anal. calcd. for $\mathrm{C}_{21} \mathrm{H}_{21} \mathrm{ClN}_{2}$ : C, 74.88; H, 6.28; N, 8.32 . Found; C, 74.64; H, 6.40; N, 8.52\%.

3-Chloro-2-phenylindeno[1,2-c]pyrazol-4(2H)-one (1b): Color: White crystals. M.p.: $144{ }^{\circ} \mathrm{C}$ (ethanol). IR (KBr, v, cm-1): $3032(\mathrm{CH})$ (Aromatic), $2963(\mathrm{CH})$ (alkyl), $1687(\mathrm{C}=0)$ (Ketone), 1590, 1485, 1455, 1430 (C=C) (Aromatic), 1385, 1260, 742. ${ }^{1} \mathrm{H}$ NMR (400 MHz, $\left.\mathrm{CDCl}_{3}, \delta, \mathrm{ppm}\right): 7.27-8.34(9 \mathrm{H}, \mathrm{m}, \mathrm{Ar}-\mathrm{H}) .{ }^{13} \mathrm{C}$ NMR (100 MHz, $\left.\mathrm{CDCl}_{3}, \delta, \mathrm{ppm}\right): 109.4$ (1C, C-3a), 122.6 (2C, Ar., C-2', C-6'), 126.8 (1C, Ar., C-4'), 127.4 (1C, Ar., C-8), 128.9 (1C, Ar., C-6), 129.4 (2C, Ar., C-3', C-5'), 130.6 (1C, Ar., C-5), 131.6 (1C, N-C(Cl)=, C-3), 135.8 (1C, Ar., C-7), 137.2 (1C, Ar., C-4a). 142.7 (1C, Ar., C-1'), 145.2 (1C, Ar., C-8a), 157.8 (1C, N=C-, C8b), 197.4 (1C, $\mathrm{C}=0$, C-4). Anal. calcd. for $\mathrm{C}_{16} \mathrm{H}_{9} \mathrm{ClN}_{2} \mathrm{O}$ : C, 68.46; H, 3.23; N, 9.98. Found; C, 68.59; H, 3.05; N, 10.18\%.

3a,4-Dihydro-2-phenyl-2H-benzo[g]indazole-3,5-dione (2c): Color: Yellow crystals. M.p.: $126{ }^{\circ} \mathrm{C}$ (ethanol). IR (KBr, v, $\left.\mathrm{cm}^{-1}\right)$ : $3035(\mathrm{CH})$ (Aromatic), $2985(\mathrm{CH})$ (alkyl), $1693(\mathrm{C}=0)$ ) (ketone),
1655 ( $\mathrm{C}=0$ ) (amide), 1580, 1480, 1465 ( $\mathrm{C}=\mathrm{C}$ ) (Aromatic), 1345, 746. ${ }^{1} \mathrm{H}$ NMR ( $\left.400 \mathrm{MHz}, \mathrm{CDCl}_{3}, \delta, \mathrm{ppm}\right): 2.71(\mathrm{dt}, 1 \mathrm{Ha}, J=14.2$ $\mathrm{Hz}, \mathrm{CH}_{2}$ ), 2.84 (dd, $1 \mathrm{H}, J=10.4 \mathrm{~Hz}, \mathrm{CH}$ ), 3.11 (app. t, $1 \mathrm{Hb}, J=$ $\left.14.2 \mathrm{~Hz}, \mathrm{CH}_{2}\right), 7.32-8.30(9 \mathrm{H}, \mathrm{m}, \mathrm{Ar}-\mathrm{H}) .{ }^{13} \mathrm{C}$ NMR $(100 \mathrm{MHz}$, $\left.\mathrm{CDCl}_{3}, \delta, \mathrm{ppm}\right): 38.6\left(1 \mathrm{C},-\mathrm{CH}_{2}-\mathrm{C}-4\right), 42.3$ (1C, C-3a), 122.8 (2C, Ar., C-2', C-6'), 126.4 (1C, Ar., C-4'), 128.3 (1C, Ar., C-6), 129.6 (2C, Ar., C-3', C-5'), 130.4 (1C, Ar., C-9), 130.9 (1C, Ar., C-9a), 132.1 (1C, Ar., C-7), 133.5 (1C, Ar., C-8), 138.7 (1C, Ar., C-5a), 145.4 (1C, Ar., C-1'), 158.7 (1C, N=C-,C-9b), 179.4 (1C, N-C=O, C3), 204.9 (1C, $\mathrm{C}=\mathrm{O}, \mathrm{C}-5$ ). Anal. calcd. for $\mathrm{C}_{17} \mathrm{H}_{12} \mathrm{~N}_{2} \mathrm{O}_{2}$ : C, 73.90; $\mathrm{H}$, 4.38; N, 10.14. Found; C, 73.76; H, 4.49; N, 10.22\%.

3a,4-Dihydro-4-methyl-2-phenyl-2H-benzo[g]indazole-3,5dione (2d): Color: White needles. M.p.: $152{ }^{\circ} \mathrm{C}$ (ethanol). IR (KBr, v, cm $\left.{ }^{-1}\right): 3066(\mathrm{CH})$ (Aromatic), $2964(\mathrm{CH})$ (alkyl), 1705 ( $\mathrm{C}=0$ ) (ketone), $1663(\mathrm{C}=0)$ (amide),1597, 1575, 1480, 1444 (C=C) (Aromatic), 1385, 743. ${ }^{1} \mathrm{H}$ NMR $\left(400 \mathrm{MHz}, \mathrm{CDCl}_{3}, \delta, \mathrm{ppm}\right)$ : $1.18\left(\mathrm{~d}, 3 \mathrm{H}, J=9.4 \mathrm{~Hz}, \mathrm{CH}_{3}\right), 2.93(\mathrm{~d}, 1 \mathrm{H}, J=12.5 \mathrm{~Hz}, \mathrm{CH}), 3.37$ $(\mathrm{p}, 1 \mathrm{H}, J=6 \mathrm{~Hz}, \mathrm{CH}), 7.23-8.41(\mathrm{~m}, 9 \mathrm{H}, \mathrm{Ar}-\mathrm{H}) .{ }^{13} \mathrm{C}$ NMR $(100$ $\left.\mathrm{MHz}, \mathrm{CDCl}_{3}, \delta, \mathrm{ppm}\right): 16.5\left(1 \mathrm{C},-\mathrm{CHCH}_{3}\right), 40.7\left(1 \mathrm{C},-\mathrm{CHCH}_{3}, \mathrm{C}-4\right)$, 46.4 (1C, C-3a), 122.6 (2C, Ar., C-2', C-6'), 126.4 (1C, Ar., C-4'), 128.5 (1C, Ar., C-6), 129.2 (2C, Ar., C-3', C-5'), 130.2 (1C, Ar., C9), 131.3 (1C, Ar., C-9a), 132.8 (1C, Ar., C-7), 134.3 (1C, Ar., C-8), 137.7 (1C, Ar., C-5a), 144.4 (1C, Ar., C-1'), 157.7 (1C, N=C-,C9b), $182.4(1 \mathrm{C}, \mathrm{N}-\mathrm{C}=0, \mathrm{C}-3), 207.2$ (1C, $\mathrm{C}=0, \mathrm{C}-5)$. Anal. calcd. for $\mathrm{C}_{18} \mathrm{H}_{14} \mathrm{~N}_{2} \mathrm{O}_{2}$ : C, 74.47; H, 4.86; N, 9.65. Found; C, 74.53; H, $5.07 ; \mathrm{N}, 9.53 \%$.

3-Chloro-4,5,6-trihydro-2-phenyl-benzo[6,7]cyclohepta[1,2c]pyrazol-6-one (3c): Color: White needles. M.p.: $91{ }^{\circ} \mathrm{C}$ (acetone). IR (KBr, v, cm-1): $3070(\mathrm{CH})$ (Aromatic), $2978(\mathrm{CH})$ (alkyl), $1694 \quad(\mathrm{C}=0)$ (ketone), 1585, 1463, $1450 \quad(\mathrm{C}=\mathrm{C})$ (Aromatic), 1385, 1280, 1075, 745. ${ }^{1} \mathrm{H}$ NMR $\left(400 \mathrm{MHz}, \mathrm{CDCl}_{3}, \delta\right.$, ppm): $1.62\left(\mathrm{t}, 2 \mathrm{H}, J=6.2 \mathrm{~Hz}, \mathrm{CH}_{2}\right), 2.84\left(\mathrm{t}, 2 \mathrm{H}, J=6.2 \mathrm{~Hz}, \mathrm{CH}_{2}\right)$, 7.19-8.30 (m, 9H, Ar-H). ${ }^{13} \mathrm{C}$ NMR $\left(100 \mathrm{MHz}, \mathrm{CDCl}_{3}, \delta, \mathrm{ppm}\right)$ : 13.8 (1C, - $\left.\mathrm{CH}_{2-}^{-}, \mathrm{C}-4\right), 52.4$ (1C, - $\left.\mathrm{CH}_{2^{-}}, \mathrm{C}-5\right), 119.3$ (1C, C-3a), 123.2 (2C, Ar., C-2', C-6'), 126.8 (1C, Ar., C-4'), 127.4 (1C, N$\mathrm{C}(\mathrm{Cl})=, \mathrm{C}-3$ ), 127.6 (1C, Ar., C-10), 128.2 (1C, Ar., C-8), 129.6 (1C, Ar., C-7), 130.4 (2C, Ar., C-3', C-5'), 132.7 (1C, Ar., C-10a), 135.7 (1C, Ar., C-9), 138.9 (1C, C-6a), 143.7 (1C, Ar., C-1'), 156.4 (1C, N=C-, C-10b), 208.3 (1C, C=0, C-6). Anal. calcd. for $\mathrm{C}_{18} \mathrm{H}_{13} \mathrm{ClN}_{2} \mathrm{O}$ : C, 70.02; H, 4.24; N, 9.07. Found; C, 69.79; H, 4.42; $\mathrm{N}, 9.35 \%$.

3-Chloro-4,5,6-trihydro-5-methyl-2-phenyl-benzo[6,7]cyclo hepta[1,2-c]pyrazol-6-one (3d): Color: White needles. 


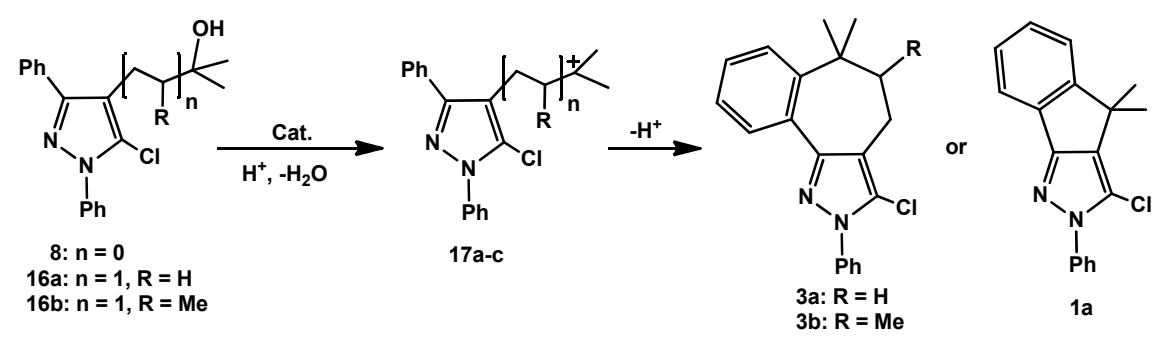

Scheme 3

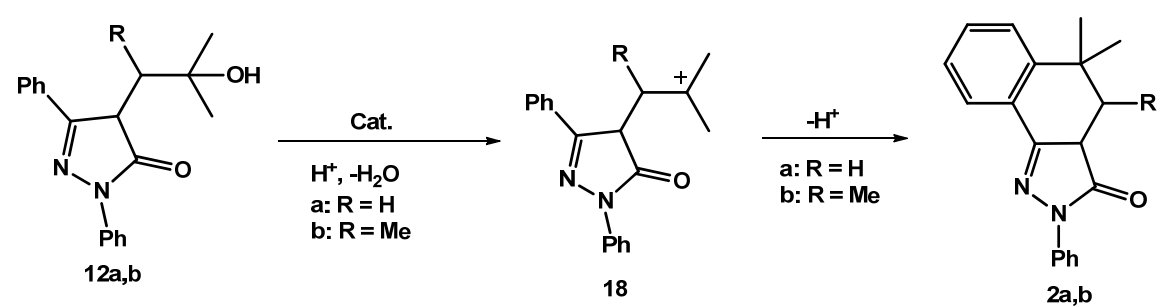

Scheme 4

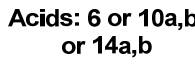
or $14 a, b$

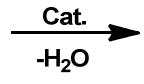<smiles>O=C1c2ccccc2-c2nn(-c3ccccc3)c(Cl)c21</smiles>

$1 b$
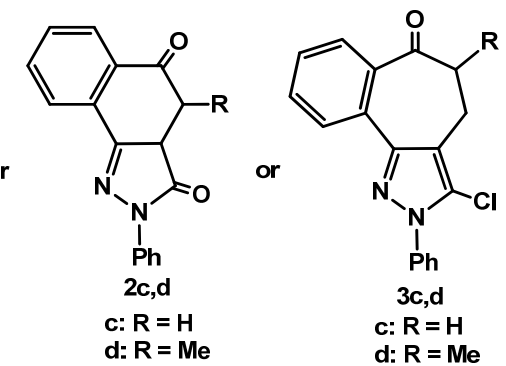

Scheme 5

M.p.: $164{ }^{\circ} \mathrm{C}$ (acetone). IR ( $\left.\mathrm{KBr}, \quad v, \mathrm{~cm}^{-1}\right): 3060(\mathrm{CH})$ (Aromatic), 2965 (CH) (alkyl), 1686 (C=0) (ester), 1590, 1470, 1450 (C=C) (Aromatic), 1385, 1285, 1070, 750, 695. ${ }^{1} \mathrm{H}$ NMR ( $\left.400 \mathrm{MHz}, \mathrm{CDCl}_{3}, \delta, \mathrm{ppm}\right): 1.19\left(\mathrm{~d}, 3 \mathrm{H}, J=10.4 \mathrm{~Hz}, \mathrm{CH}_{3}\right), 2.64$ (td, $\mathrm{Ha}, J=16.3 \mathrm{~Hz}, \mathrm{CH}_{2}$ ), 2.81 (p, $\mathrm{Hb}, J=16.3 \mathrm{~Hz}, \mathrm{CH}_{2}$ ), 3.42 (q, $1 \mathrm{H}, J=9.3 \mathrm{~Hz},-\mathrm{CH}), 7.30-8.35(\mathrm{~m}, 9 \mathrm{H}, \mathrm{Ar}-\mathrm{H}) .{ }^{13} \mathrm{C}$ NMR $(100 \mathrm{MHz}$, $\mathrm{CDCl}_{3}, \delta, \mathrm{ppm}$ ): 19.4 (1C, $\left.-\mathrm{CHCH}_{3}\right), 29.5$ (1C, $\left.-\mathrm{CH}_{2}-, \mathrm{C}-4\right), 47.2$ (1C, $\left.-\mathrm{CHCH}_{3}, \mathrm{C}-5\right), 118.5$ (1C, C-3a), 123.4 (2C, Ar., C-2', C-6'), 126.8 (1C, Ar., C-4'), 127.2 (1C, N-C(Cl)=, C-3), 128.2 (1C, Ar., C10), 128.9 (1C, Ar., C-8), 129.2 (1C, Ar., C-7), 130.3 (2C, Ar., C-3', C-5'), 131.7 (1C, Ar., C-10a), 134.7 (1C, Ar., C-9), 138.9 (1C, Ar., C-6a), 144.5 (1C, Ar., C-1'), 157.3 (1C, N=C-, C-10b), 208.2 (1C, $\mathrm{C}=0$, C-6). Anal. calcd. for $\mathrm{C}_{19} \mathrm{H}_{15} \mathrm{ClN}_{2} \mathrm{O}: \mathrm{C}, 70.70 ; \mathrm{H}, 4.68 ; \mathrm{N}, 8.68$. Found; C, 70.92; H, 4.45; N, 8.52\%.

\section{Results and discussion}

We first attempted to prepare the precursors heteroaryl acids required for this work $(\mathbf{6}, \mathbf{1 0 a}, \mathbf{b}$ and $14 a, b)$ which are easily obtained via three different synthetic routes starting from the easily prepared 1,3-diphenyl-1H-pyrazol-5(4H)-one (4) [56] as formulated in Scheme 1 and 2.

The first path comprised the formation of 5-chloro-1,3diphenyl-1H-pyrazole-4-carbaldehyde (5) following standard literature procedure by chloroformylation of pyrazolone $\mathbf{4}$ under Vilsmeier conditions [55]. This aldehyde was oxidized by $\mathrm{KMnO}_{4}$ solution [57] to afford 5-chloro-1,3-diphenyl- $1 \mathrm{H}$ pyrazole-4-carboxylic acid (6). The second path included the conversion of substrate 4 to 4-bromo-1,3-diphenyl-1H-pyrazol-
$5(4 H)$-one (9) following the literature procedure [58] in the presence of bromine in acetic acid. The resulting bromide 9 was allowed to react with excess of sodio-diethyl malonates [59] in ethanol followed by decarboxylation to afford substituted acetic acids 10a,b.

On the other hand, the third route (path 3 ) involved the production of acid precursors 14a,b (Scheme 2). These acids were smoothly prepared through the Perkin-type reaction [60] of aldehyde $\mathbf{5}$ with acid anhydrides and the corresponding sodium salts of acids to give substituted 3-(5-chloro-1,3diphenyl-1 $H$-pyrazol-4-yl)acrylic acids $(\mathbf{1 1 a}, \mathbf{b})$. The resulting acids 13a,b were reduced by sodium amalgam in sodium hydroxide solution to afford 14a,b.

Upon intermediate acids $\mathbf{6}, \mathbf{1 0 a}, \mathbf{b}$ and $\mathbf{1 4 a}, \mathbf{b}$ are formed, they could be converted into ethyl esters $7, \mathbf{1 1 a}, \mathbf{b}$ and $\mathbf{1 5 a}, \mathbf{b}$ by reaction with ethanol and $\mathrm{H}_{2} \mathrm{SO}_{4}$ [61]. The resulting esters were converted to the corresponding alcohols 8, 12a,b and 16a,b (Schemes 1 and 2; Table 1) by addition of two equivalents of methymagnesium iodide [62] in $\mathrm{Et}_{2} \mathrm{O} / \mathrm{THF}$ solution. The structures of all new alcohols were appropriately established by the usual spectroscopic methods.

The structural elucidation of alcohols $8,12 a, b$, and $16 \mathbf{a}, \mathbf{b}$ was mainly based on IR, ${ }^{1} \mathrm{H}$ NMR, MS and elemental analyses. The IR spectra of alcohols 1a-c showed absorption bands for a $\mathrm{OH}$ groups as broad bands in the range $3340-3490 \mathrm{~cm}^{-1}$. The ${ }^{1} \mathrm{H}$ NMR data allowed an unambiguous statement of the heterocyclic alkanols formation. Thus, the ${ }^{1} \mathrm{H}$ NMR spectrum displayed five signals for compound 12a. The tenth aromatic protons appear at $\delta$ 7.15-8.25 ppm. 

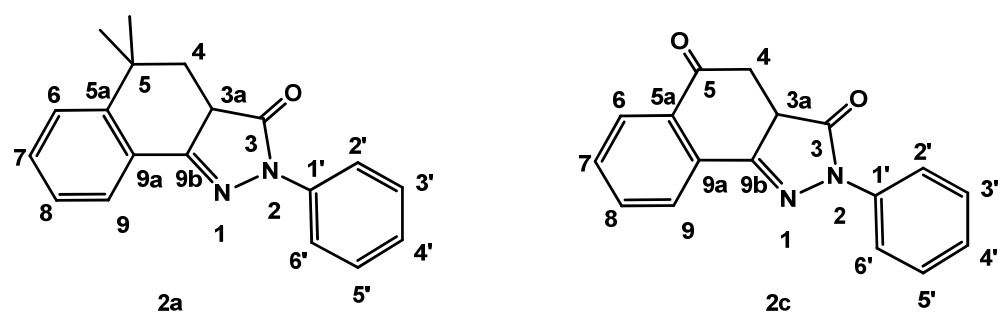

Figure 2. Diastereotopic protons embraced in pyrazolones heteropolycycles.

The aliphatic acyclic protons of the two methyls and methylene groups of the new alcohol appeared as two sets of splitting. The singlet gem-dimethyls appear at $\delta 1.30 \mathrm{ppm}$ while doublet at $\delta 2.0 \mathrm{ppm}$ was assigned to the up-field proton $\mathrm{CH}_{2}$. A forth broad singlet at $\delta 2.55 \mathrm{ppm}$ was assigned to $\mathrm{OH}$. In all IR spectra of the synthesized carbinols, the characteristic peak of carbonyl groups was absent.

The cyclialkylations of alcohols 8, 12a,b and 16a,b were smoothly carried out in the presence of $\mathrm{AlCl}_{3} / \mathrm{CH}_{3} \mathrm{NO}_{2}$ or $\mathrm{P}_{2} \mathrm{O}_{5}$ or PPA catalysts under different reaction conditions gave substituted 2,4-dihydroindeno[1,2-c]pyrazole 1a, 4,5-dihydro$2 H$-benzo[ $g]$ indazol-3(3aH)-ones $\mathbf{2 a , b}$ and 4,5,6-trihydro benzo[6,7]cyclohepta[1,2-c]pyrazoles $\mathbf{3 a , b}$ in good overall yields (Table 2, Scheme 3 and 4).

On the other hand, cyclialkylations of acids 6, 10a,b and 14a,b were carried out in the presence of the same catalysts under varying conditions to give substituted tricyclic pyrazolones $\mathbf{1 b}, \mathbf{2 c , \mathbf { d }}$ and $\mathbf{3 c , d}$. The results are embedded in Table 2 and Scheme 5.

The ${ }^{1} \mathrm{H}$ NMR data allowed an unambiguous statement of the formation of heterocyclic products. The assignment of the diastereotopic protons of the methylene group directly bonded to pyrazole or pyrazolone rings $(\mathbf{2 a}, \mathbf{c}$ and $\mathbf{3 b} \mathbf{b}, \mathbf{d})$ beside their intermediates alcohols and acids (12a, 16b and 10a, 14b) were made on the basis of $J$-values that were obtained by direct inspection of the ${ }^{1} \mathrm{H}$ NMR spectrum which in turn showed a greatly change in chemical shifts. It is known that the extent of shielding is proportional to the electronegativity exerted by the heterocyclic ring or the neighbored functional groups and its proximity to the hydrogen [63-66].

This trend has been observed from the chemical shifts of diastereotopic hydrogens $\left(\mathrm{H}_{a}\right.$ and $\left.\mathrm{H}_{b}\right)$ of $\mathrm{CH}_{2}$ group characterized by the $\mathrm{A}_{2} \mathrm{~B}$ system obtained in each series (Figure 2).

For instance, the ${ }^{1} \mathrm{H}$ NMR of tricyclic compound 2a displayed five signals in which aromatic protons appeared at $\delta$ $6.85-8.11 \mathrm{ppm}$ and the gem-dimethyls groups appeared as singlet at $\delta 1.40 \mathrm{ppm}$, respectively. The third quartet appeared at $2.35 \mathrm{ppm}$ with coupling constant of $10.4 \mathrm{~Hz}$. As expected, the remaining two signals for the diastereotopic $\mathrm{CH}_{2}$ group exhibits a complex set of overlapped signals which were assigned to be shielded and displayed as a second order set of signals showed a triplet in average at $\delta 1.84 \mathrm{ppm}$ with coupling constant of $14.2 \mathrm{~Hz}$ for one hydrogen and doublet of doublets at an average of $2.17 \mathrm{ppm}$ for the other one. In comparison with the compound 2a, the tricyclic pyrazolone $2 \mathrm{c}$ showed the ${ }^{1} \mathrm{H}$ NMR chemical shifts for $\mathrm{CH}_{2}$ protons $\left(\mathrm{H}_{a}\right.$ and $\left.\mathrm{H}_{b}\right)$ as a characteristic doublet of triplet for $\mathrm{H} a$ at an average of $\delta 2.71 \mathrm{ppm}$ with a coupling constant of $14.2 \mathrm{~Hz}$ and a apparently triplet for $\mathrm{H}_{b}$ near $\delta 3.11 \mathrm{ppm}$.

\section{Conclusion}

In conclusion, we have developed a simple and attractive method for the synthesis of variety of new fused tricyclic pyrazoles bearing small to medium-sized alkyl residues. The synthetic approach involved Friedel-Crafts cyclialkylations of heteroaryl alkanols and acids catalyzed by $\mathrm{AlCl}_{3} / \mathrm{CH}_{3} \mathrm{NO}_{2}$ or $\mathrm{P}_{2} \mathrm{O}_{5}$ or PPA. To the best of our knowledge, this is the first time that such novel substituted pyrazoles have been described. The results proved that Friedel-Crafts cyclialkylations can be considered as useful pathways to the syntheses of heteropolycycles.

\section{Acknowledgements}

The authors are grateful for the financial support and all the facilities received while performing and writing this work by the Chemistry Department, Faculty of Science Assiut University, Assiut, Egypt.

\section{References}

[1]. Kleemann, A.; Engel, J.; Kutscher, B.; Reichert, D. Pharmaceutical Substances, 3rd edition.; Georg Thieme Verlag: Stuttgart/New York, 1999, p 1725-1727.

[2]. Deng, X.; Mani, N. S. Org. Lett. 2008, 10, 1307-1310.

[3]. Bhat, B. A.; Dhar, K. L.; Puri, S. C.; Saxena, A. K.; Shanmugavel, M.; Qazi, G. N. Bioorg. Med. Chem. Lett. 2005, 15, 3177-3180.

[4]. Gandhale, D. N.; Patil, A. S.; Awate, B. G.; Naik, L. M. Pesticides 1982, 16, 27-28.

[5]. Elguero, J.; Goya, P.; Jagerovic, N.; Silva, A. M. S. Pyrazoles as Drugs: Facts and Fantasies. In Targets in Heterocyclic Systems, Vol. 6, Attanasi, 0. A.; Spinelli, D. Eds., Italian Society of Chemistry, Roma, Italy, 2002, pp. 52-98.

[6]. Ishii, T.; Shimortori, H.; Tanaka, Y.; Ishikawa, K. Japan Patent, JP 01, 168, 675 Chem. Abstr., 1989, 112, 35854.

[7]. Robin, E. M.; David, R. G.; Vijayalekshmi, S. Phytochemistry 2008, 69, 2704-2707.

[8]. Karthikeyan, M. S.; Holla, B. S.; Kumari, N. S. Eur. J. Med. Chem. 2007, $42,30-36$.

[9]. Gokhan-Kelekci, N.; Yabanoglu, S.; Kupeli, E.; Salgin, U.; Ozgen, O.; Ucar, G.; Yesilada, E.; Kendi, E.; Yesilada, A.; Bilgin, A. A. Bioorg. Med. Chem. 2007, 15, 5775-5786.

[10]. Xia, Y.; Fan, C. -D.; Zhao, B. -X.; Zhao, J.; Shin, D. -S.; Miao, J. Y. Eur. J. Med. Chem. 2008, 43, 2347-2353.

[11]. Huang, Y. R.; Katzenellenbogen, J. A. Org. Lett. 2000, 2, 2833-2836.

[12]. Sarojini, B. K.; Vidyagayatri, M.; Darshanraj, C. G.; Bharath, B. R.; Manjunatha, H. Lett. Drug Des. Disc. 2010, 7, 214-224.

[13]. Behr, L. C.; Fusco, R.; Jarboe, C. H. In The Chemistry of Heterocyclic Compounds, Pyrazoles, Pyrazolines, Pyrazolidines, Indazoles and Condensed Rings" Weissberger, A. , Ed.; Interscience Publishers: New York, 1967, pp. 1-72.

[14]. Mahajan, N.; Havaldar, F. H.; Fernandes, P. S. J. Indian Chem. Soc. 1991, $68,245-248$.

[15]. Grosche, P.; Holtzel, A.; Walk, T. B.; Trautwein, A. W.; Jung, G. Synthesis 1999, 1961-1970.

[16]. Bailey, D. M.; Hansen, P. E.; Hlavac, A. G.; Baizman, E. R.; Pearl, J.; Defelice, A. F.; Feigenson, M. E. , J. Med. Chem. 1985, 28, 256-260.

[17]. Kurowaski, M.; Dunky, A.; Geddawi, M. Eur. J. Clin. Pharmacol. 1987, 307, 31-34.

[18]. Said, A. S.; Abd El-Galil, E. A.; Nermien, M. S.; Mohamed, M. Eur. J. Med. Chem. 2009, 44, 4787-4792.

[19]. Barcelo, M.; Ravina, E.; Masaguer, C. F.; Dominuez, E.; Areias, F. M. Brea, J.; Loza, M. I. . Bioorg. Med. Chem. Lett. 2007, 17, 4873-4877.

[20]. Chou, L. -C.; Huang, L. -J.; Yang, J. -S.; Lee, F. -Y.; Teng, C. -M.; Kuo, S. -C. Bioorg. Med. Chem. 2007, 15, 1732-1740.

[21]. Narasimhulu, B. N.; Jacques, B.; Francis, B.; Timothy, P. C.; Mark, R. K.; John, D. M.; Carl, O.; Serge, P.; Roger, R.; Margaret, E. S.; Yasutsugu, U.; Michael, A. W. United States Patent 7511037, US 7, 511, 037B2, 2009.

[22]. Moser, H.; Boehner, B.; Foery, W.; European Patent, EP 268, 554, Chem. Abstr., 1988, 110, 23879. 
[23]. Natsume, B.; Kyomura, N.; Kikutake, K.; Fukuch, T. Eur. Pat. Appl. EP., 462, 573, Chem. Abstr., 1992, 116, 128

[24]. Windholz, M. The Merck Index, 9th edition, Merck and Co., Rahway: New Jersey, 1976.

[25]. Kim, M.; Sim, C.; Shin, D.; Suh, E.; Cho, K. Crop Protect 2006, 25, 542548.

[26]. Fahmy, S. M.; Badran, A. H.; Elnagdi, M. H. J. Chem Tech. B: Technol. 1980, 30, 390-395.

[27]. Eicher, T.; Hauptmann, S. The Chemistry of Heterocycles: Structure, Reactions, Syntheses, and Applications, $2^{\text {nd }}$ Edition, Wiley-VCH, ISBN 3527307206, 2003.

[28]. Knorr, L. J. Ann. Chem. 1894, 379, 236-242.

[29]. Aggarwal, V. K.; Vicente, D. V; Bonnert, R. V. J. Org. Chem. 2003, 68, 5381-5383.

[30]. Elguero, J. Comprehensive Heterocyclic Chemistry, Vol. 5, Katritzky, A. R.; Rees, C. W. Eds., Pergamon Press, Oxford, 1984, pp. 167-303.

[31]. Deng, X.; Mani, N. S. Org. Lett. 2006, 8, 3505-3508.

[32]. Noguchi, M.; Sunagawa, T.; Akao, R.; Yamada, H.; Yamamoto, H.; Kakehi, A. Tetrahedron 2007, 63, 4548-4557.

[33]. Bhat, B. A.; Puri, S. C.; Qurishi, M. A.; Dhar, K. L.; Qazi, G. N. Synth. Commun. 2005, 35, 1135-1142.

[34]. Almazroa, S.; Elnagdi, M. H.; Salah El-Din, A. M. J. Heterocycl. Chem. 2004, 41, 267-272.

[35]. Fustero, S.; Roman, R.; Sanz-Cervera, J. F.; Simon-Fuentes, A. X.; Cunat, A. C.; Villanova, S.; Murguia, M. J. Org. Chem. 2008, 73, 3523-3529.

[36]. Ahmed, M. S. M.; Kobayashi, K.; Mori, A. Org. Lett. 2005, 7, 4487-4489.

[37]. Sherif, A.; Rostom, F. Bioorg. Med. Chem. 2006, 14, 6475-6485.

[38]. Shi, C. L.; Shi, D. Q.; Kim, S. H.; Huang, Z. B.; Ji, S. J.; Ji, M. Tetrahedron, 2008, 64, 2425-2432.

[39]. Jagtap, P. G.; Degterev, A.; Choi, S.; Keys, H.; Yuan, J.; Cuny, G. D. J. Med. Chem. 2007, 50, 1886-1895.

[40]. El-Rayyes, N. R.; Al-Jawhary, A. J. Heterocycl. Chem. 1986, 23, 135-140.

[41]. Klimova, E. I.; Klimova, T.; Garcia, M. M.; Vazquez Lopez, E. A.; Esparza, R. M.; Alvarado Rodriguez, J. G.; Ramirez Apan, T. J. Organomet. Chem. 2003, 665, 69-75.

[42]. Bergemann, S.; Brecht, R.; Büttner, F.; Guenard, D.; Gust, R.; Seitz, G.; Stubbs, M. T.; Thoret, S. , Bioorg. Med. Chem. 2003, 11, 1269-1281.

[43]. Boolell, M.; Gepi-Attee, S.; Gingell, J. C.; Allen, M. J. Br. J. Urol. 1996, 78, 257-261.

[44]. Pacher, P.; Nivorozhkin, A.; Szabó, C. Pharmacol. Rev. 2006, 58, 87 114.

[45]. Li, A.; Gilbert, T. M.; Klumpp, D. A. J. Org. Chem. 2008, 73, 3654-3657.

[46]. Khalaf, A. A.; El-Khawaga, A. M.; Awad, I. M.; Abd El-Aal, H. A. K. Arkivoc 2010, 10, 338-349.

[47]. Abd El-Aal, H. A. K.; Khalaf, A. A.; Arkivoc 2013, 4, 306-322.

[48]. Abd El-Aal, H. A. K.; Khalaf, A. A. Aust. J. Chem., 2013, 66, 635-645.

[49]. Khalaf, A. A.; El-Khawaga, A. M.; Awad, I. M.; Abd El-Aal, H. A. K. Arkivoc 2009, 14, 314-323.

[50]. Khalaf, A. A.; El-Khawaga, A. M.; Awad, I. M.; Abd El-Aal, H. A. K. Arkivoc 2010, 10, 338-349.

[51]. Olah, G. A. In Friedel-Crafts Chemistry; Olah, G. A. , Ed.; Wiley: New York, NY, 1973.

[52]. Barclay, L. R. C. In Friedel-Crafts and Related Reactions, Vol. II, Olah, G. A. Ed.; Interscience, New York, 1964, Chap. 22 and references therein.

[53]. Roberts, R. M.; Khalaf, A. A. Friedel-Crafts Chemistry: A Century of Discovery, Marcel Dekker: New York, NY, 1984.

[54]. Poulsen, T. B.; Jørgensen, K. A. Chem. Rev. 2008, 108, 2903-2915.

[55]. El-Shekeil, A.; Babaqi, A.; Hassan, M.; Shiba, S. Heterocycles 1988, 27, 2577-8250.

[56]. Li, J. Y.; Wang, X. Y.; Zhao, Q. H. Chem. Reag. 1997, 19, 112-117.

[57]. Datterl, B.; Trostner, N.; Kucharski, D.; Holzer, W. Molecules 2010, 15, 6106-6126.

[58]. Smith, L. Kgl. Fysiograf. Sällskap. Lun., Förh. 1948, 18, 3-5. Chem. Abstr., 1950, 44, 1949e.

[59]. Bachmann, W. E. and Sheehan, J. C. , J. Am. Chem. Soc. , 1941, 63, 204206.

[60]. Li, L.; Elliott, W. H. J. Am. Chem. Soc. 1952, 74, 4089-4090.

[61]. Jones, R. G.; Soper, Q. F.; Behrens, O. K.; Corse, J. W. J. Am. Chem. Soc. 1948, 70, 2843-2848.

[62]. Perold, G. W.; Steyn, A. P.; Von Reiche, F. V. K. J. Am. Chem. Soc. 1957, 79, 462-465.

[63]. Hesse, M.; Meier, H.; Zeeh, B. Spektroskopische Methoden in der Organischen Chemie, $4^{\text {th }}$ edition, Thieme Verlag: New York, 1991.

[64]. Bonacorso, H. G.; Correa, M. S.; Porte, L. M. F.; Pittaluga, E. P.; Zanatta, N.; Martins, M. A. P. Tetrahedron Lett. 2012, 53, 5488-5491.

[65]. Becker, E. High Resolution NMR Theory and Chemical Application, 3rd edition, Academic Press, 2000, pp 139-181.

[66]. Silverstein, R. M.; Webster, F. X.; Kiemle, D. J. Spectrometric Identification of Organic Compounds, $7^{\text {th }}$ edition, Wiley, Hoboken, 2005. 